\title{
Spontaneous activity generated within the olfactory bulb establishes the discrete wiring of mitral cell dendrites
}

\author{
Satoshi Fujimoto ${ }^{1,2,8}$, Marcus N. Leiwe ${ }^{1,2,8}$, Richi Sakaguchi ${ }^{1,2,3}$, Yuko \\ Muroyama $^{4}$, Reiko Kobayakawa ${ }^{5}$, Ko Kobayakawa ${ }^{5}$, Tetsuichiro Saito ${ }^{4}, \&$ \\ Takeshi Imai ${ }^{1,2,3,6,7}$ \\ ${ }^{1}$ Graduate School of Medical Sciences, Kyushu University, Fukuoka 812-8582, Japan. ${ }^{2}$ \\ Laboratory for Sensory Circuit Formation, RIKEN Center for Developmental Biology, Kobe \\ 650-0047, Japan. ${ }^{3}$ Graduate School of Biostudies, Kyoto University, Kyoto 606-8501, Japan. ${ }^{4}$ \\ Department of Developmental Biology, Graduate School of Medicine, Chiba University, Chiba \\ 260-8670, Japan. ${ }^{5}$ Institute of Biomedical Science, Kansai Medical University, Hirakata 573- \\ 1010, Japan. ${ }^{6}$ PRESTO, Japan Science and Technology Agency (JST), Saitama 332-0012, Japan. \\ ${ }^{7}$ Research and Development Center for Five-Sense Devices, Kyushu University, Fukuoka 819- \\ 0395, Japan. ${ }^{8}$ These authors contributed equally to this work. \\ Correspondence should be addressed to T.I. (t-imai@med.kyushu-u.ac.jp).
}

\begin{abstract}
In the mouse olfactory bulb, sensory information detected by 1,000 types of olfactory sensory neurons (OSNs) is represented by the glomerular map. The second-order neurons, mitral and tufted cells, connect a single primary dendrite to one glomerulus. This forms discrete connectivity between the $\mathbf{1 , 0 0 0}$ types of input and output neurons. It has remained unknown how this discrete dendrite wiring is established during development. We found that genetically silencing neuronal activity in mitral cells, but not from OSNs, perturbs the dendrite pruning of mitral cells. In vivo calcium imaging of awake neonatal animals revealed two types of spontaneous neuronal activity in mitral/tufted cells, but not in OSNs. Pharmacological and knockout experiments revealed a role for glutamate and NMDARs. The genetic blockade of neurotransmission among mitral/tufted cells reduced spontaneous activity and perturbed dendrite wiring. Thus, spontaneous network activity generated within the olfactory bulb self-organizes the parallel discrete connections in the mouse olfactory system.
\end{abstract}

\section{INTRODUCTION}

Sensory information detected by sensory organs is represented by spatiotemporal patterns of 
activity in corresponding regions of the brain. In general, continuous physical information is spatially represented by continuous topographic maps, such as the retinotopic map for visual space and the tonotopic map for sound frequencies. The formation of continuous maps and their connectivity is known to be regulated by a combination of graded guidance cues and neuronal activity $^{1,2}$. The coarse targeting of axons is controlled by the graded expression of guidance molecules such as ephrins and Eph receptors ${ }^{3}$. Later on, spontaneous activity generated by the sensory organ (e.g., retina and cochlea) fine-tunes connections to produce a precise nearestneighbour relationship from sensory to higher-order neurons ${ }^{1,4-8}$. In vivo imaging of the neonatal mouse brain demonstrated a propagating wave of activity originating from the retina into the entire visual system in the brain ${ }^{9}$. Similarly, spontaneous activity generated in the developing cochlea propagates all the way to the auditory brain regions ${ }^{10}$.

In contrast, the olfactory system has to detect a vast variety of odorants, which are mostly discrete in nature and convey distinct information. Odorants are detected by a large family of odorant receptors ( $\sim 0$ in insects and $\sim 1,000$ in mammals) which are expressed by olfactory sensory neurons (OSNs) ${ }^{11}$. OSNs expressing the same type of odorant receptor converge their axons onto one or a few glomeruli in the olfactory bulb. As a result, olfactory information is represented by spatiotemporal patterns of activity in the glomerular map ${ }^{12,13}$. In mammals, sensory input into one glomerulus is relayed to 20-50 mitral and tufted cells, which project their axons to the olfactory cortices. Each mitral/tufted cell receives inputs from just one glomerulus, allowing for segregated odor information processing. A glomerulus is also innervated by various types of interneurons and centrifugal inputs, which perform dynamic odor tuning including gain control, lateral inhibition, pattern separation, and oscillatory activity for phase coding ${ }^{14,15}$. Thus, the segregated glomerular microcircuitry is an important platform for odor information processing and for subsequent behaviors.

Over the last two decades, the mechanisms of neuronal wiring specificity in the glomerular map have been intensively studied in Drosophila and mice. In the Drosophila olfactory system, the connectivity of OSN axons and second-order neurons is mostly geneticallydetermined with a variety of transcription factors and cell-surface molecules ${ }^{16,17}$. Notably, the wiring specificity in the Drosophila olfactory system is based on molecular matching between pre- and post-synaptic neurons ${ }^{18,19}$.

However, the wiring of the mouse olfactory system is more flexible in order to accommodate a much larger number of odorant receptors. For example, the ectopic expression of a new receptor leads to the formation of a new glomerular microcircuit in the olfactory bulb ${ }^{20}$, refuting the possibility of a strictly genetically-determined regulation. It has been revealed that the axonal projections of OSNs are regulated by a combination of genetic programs, odorant receptor-derived cAMP signals, and neuronal activity, which together define the combinatorial expression of various axon guidance molecules ${ }^{12,21-26}$. Notably, the glomerular map is mostly formed by axon-axon interactions. However, these mechanisms are merely one side of the coin: 
The dendrite wiring of mitral/tufted cells also needs to be precisely controlled. It has been known that migration and the domain organization of mitral/tufted cells within the olfactory bulb is genetically-determined ${ }^{27-29}$. Later on, the discrete dendrite wiring of each mitral cell to a glomerulus is established through a dendrite remodeling process. While mitral cells initially extend multiple dendrites, just one of them is strengthened to form a mature primary dendrite and the supernumerary ones are pruned during development ${ }^{30}$. Activity-dependence has been an important issue, in relation to other sensory systems ${ }^{31}$; however, previous efforts have all failed to find a contribution for OSN-derived neuronal activity in dendrite remodeling ${ }^{32-34}$. Therefore, it has long been an enigma as to how the discrete wiring of mitral/tufted cell dendrites is established in the mammalian olfactory system ${ }^{14}$. In the present study, we found that the dendrite pruning of mitral cells is in fact an activity-dependent process. Unlike other sensory systems, spontaneous activity is generated "within the olfactory bulb" and "self-organizes" the discrete connectivity of mitral cell dendrites.

\section{RESULTS}

\section{Developmental remodeling of mitral cell dendrites}

To understand mechanisms of dendrite wiring in mitral cells, we took a genetic approach using in utero electroporation ${ }^{35-37}$. It has been known that mitral cells are generated between embryonic day (E) 10 and E13 ${ }^{29,38}$. Therefore, we electroporated plasmid DNA at E12 to label a subset of mitral cells (Fig. 1b). To facilitate the reconstruction of fine neuronal morphology, we electroporated a Cre-dependent tdTomato plasmid and a small amount of Cre plasmid, which produced sparse and bright labeling (Fig. 1a). To avoid any biases in quantification, we obtained volumetric fluorescence image data from olfactory bulb tissues cleared with SeeDB or SeeDB2 39,40 , and analyzed mitral cells in the medial side of the olfactory bulb in an unbiased manner (Supplementary Fig. 1 and Supplementary Video 1). We only focused on labelled neurons in the mitral cell layer, and excluded tufted cells from our analyses.

A typical mitral cell has a single primary dendrite (also known as an apical dendrite) connecting one glomerulus and several lateral dendrites (also known as basal dendrites) extending within the external plexiform layer. During development, mitral cells initially extend multiple dendrites toward the glomerular layer ${ }^{30,32,38,41}$. At postnatal day 1 (P1), the labelled mitral cells extend $8.4 \pm 2.4$ (mean \pm SD) primary dendrites to the glomerular layer (Fig. 1c,d) and $85 \%$ of these cells did not have a tufted structure at the end of any dendrite (Fig. 1e,f). By P3, the number of primary dendrites has decreased to $4.0 \pm 1.0$ (mean \pm SD, Fig. 1c,d) while the number of cells containing at least one dendrite with a ramified tufted structure increased to $67.1 \%$, with $30.2 \%$ containing two or more (Fig. 1f). We also examined the formation of synapses using PSD 1 1.2-GFP, which labels mature glutamatergic synapses without affecting synapse formation ${ }^{42,43}$. Prominent PSD $\Delta 1$.2-GFP puncta began to appear in tufted structures 
from P3 (Fig. 1e). Finally, at P6 most mitral cells have a single primary dendrite extending to a single (1.4 \pm 0.7, mean \pm SD) glomerulus (Fig. 1d), with $79.7 \%$ of cells containing a single tufted dendrite. Day-to-day quantification indicated that the pruning of supernumerary dendrites occurs between P4-5 (Supplementary Fig. 1c). The formation of lateral dendrites also occurred during P3-6.

Together, these observations indicate that the remodeling of mitral cell dendrites occurs in a step-wise manner (Fig. 1g). During Phase I $(\leq \mathrm{P} 3)$, the number of dendrites is gradually reduced to $\sim 4$ and a tufted structure with excitatory synapses begins to appear in some of them. During Phase II (P3-6), just one dendrite is further strengthened to have a ramified tufted structure, while the others are pruned. Several lateral dendrites are also formed during this stage. Thus, dendrite pruning and synapse elimination occurs during Phase II at least in a subset of mitral cells.

\section{Neuronal activity in mitral cells is essential to establish a single primary dendrite}

Neuronal activity often plays an important role in neuronal remodeling in the mammalian brain ${ }^{44}$. However, previous attempts to determine a role for neuronal activity in mitral cell remodeling have been unsuccessful ${ }^{32-34,41}$. As all of these studies manipulated neuronal activity only in OSNs, we directly manipulated neuronal activity in mitral cells by overexpressing a gene coding for the inward rectifying potassium channel, Kir2.1 ${ }^{45}$. Overexpression of Kir2.1 efficiently inhibited the neuronal activity of mitral cells in a cell-autonomous fashion (Supplementary Fig. 2a). Kir2.1 overexpression did not affect the initial growth (P1) and remodeling of dendrites at the early stage (Phase I). However, during Phase II, dendrite pruning was specifically perturbed (Fig. 2a,b). In control neurons, 78.1\% of mitral cells connected to a single glomerulus at P6; however, only $19.3 \%$ of Kir2.1-expressing neurons connected to a single glomerulus at this stage. Even by P28, half of the mitral cells still connected to multiple glomeruli (Fig. 2b). The formation of lateral dendrites was also precluded (Fig. 2c). In contrast, the formation of mitral cell axons was not affected at P6 (Supplementary Fig. 2b). Tufted structures and PSD $\Delta 1.2-G F P$ puncta were still visible in the primary dendrites of Kir2.1-expressing mitral cells, suggesting synapse formation (Fig. 2e). Thus, the pruning of supernumerary dendrites and the formation of lateral dendrites, which normally occur during Phase II, were specifically perturbed by Kir2.1 overexpression. Defective dendrite pruning is due to the hyperpolarizing effects of Kir2.1, because a non-conducting mutant of Kir2.1 ${ }^{45}$ did not affect the pruning process (Supplementary Fig. 2c). Overexpression of the bacterial sodium channel NaChBac which increases the excitability of neurons did not affect the dendrite remodeling process (Supplementary Fig. 2d) ${ }^{46}$, although radial migration of the cell body was affected as reported for cortical pyramidal neurons (Supplementary Fig. 2e) ${ }^{47}$.

Activity-dependent dendrite pruning is not just a specific feature of mitral cells labelled at a particular timing (E12) by in utero electroporation. We also overexpressed Kir2.1 in mitral 
cells generated earlier (in utero electroporation at E10). While these neurons potentially develop earlier than E12-labelled ones ${ }^{29}$, Kir2.1 overexpression similarly prevented their dendrite pruning (Supplementary Fig. 2f). Thus, activity-dependent dendrite pruning is a common feature between early-born and late-born mitral cells. Hereafter, we only studied mitral cells labelled at E12.

\section{Neuronal activity derived from OSNs is dispensable}

Could the neuronal activity required for pruning be derived from OSNs? Previous studies failed to show a role for neuronal activity from OSNs, but with important caveats ${ }^{32-34,41}$. This may be due to different labeling and imaging techniques, an incomplete blockade of neuronal activity, and/or retardation of animal growth. We therefore re-examined the role of neuronal activity in OSNs using our analysis pipeline (Supplementary Fig. 1a).

Firstly, we examined the role of sensory-evoked activity, i.e., odor and mechanosensory responses in OSNs ${ }^{48,49}$. Both of these sensory stimuli can be effectively eliminated by unilateral naris occlusion from P0. We found no change in primary dendrite pruning at P6 (Fig. 3a). Thus, sensory-evoked activity is dispensable for the normal development of mitral cell dendrites.

We next examined the role of activity (both evoked and spontaneous) generated by the olfactory signal transduction cascade involving cyclic nucleotide-gated (CNG) channels, as this has also been known to contribute to spontaneous activity ${ }^{22,50}$. We used the OMP-Cre knock-in line to knockout CNGA2 specifically in OSNs. In the conditional mutant, dendrite pruning was incomplete at P6, but recovered to normal levels at P14 (Fig. 3b), consistent with an earlier study using the CNGA2 straight knockout ${ }^{32}$. As this anosmic mutant animal shows severe growth retardation, likely due to poor suckling (Fig. 3c), this may explain the poor dendrite pruning. To further test the role of any kind of activity produced in OSNs, we generated another mouse line expressing tetanus toxin light chain (TeNT), which cleaves VAMP2 and thus inhibits synaptic vesicle release ${ }^{51,52}$. In OMP-Cre;R26-CAG-LoxP-TeNT (designated OSN-TeNT) mice, antiVAMP2 staining signals in OSNs were completely eliminated (Fig. 3d). OSN-TeNT mice showed defective dendrite pruning at P6, but normal dendrite morphology at P14 (Fig. 3e). Again, OSN-TeNT mice demonstrated a severe growth retardation (Fig. 3f). Therefore, we could not conclude whether this indicates a role for activity in OSNs, or just a developmental delay in this mouse line.

To clarify this issue, we tried to rescue the growth retardation by hand rearing new-born animals. OSN-TeNT mice are apparently anosmic and cannot suckle very well. Therefore, we fed powdered milk to OSN-TeNT mice manually. We fed pups every 2 hours from P1-6 in a warm chamber (Fig. 3g,h). To facilitate digestion, excretion and to prevent bloating, we also massaged their stomach after feeding. This hand rearing partially rescued the growth delay (Fig. $3 \mathbf{i})$, and these OSN-TeNT mice weighted similarly (98.9 $\pm 11.1 \%$, mean \pm SD) to hand-reared controls, and $62.2 \pm 12.9 \%$ (mean $\pm \mathrm{SD}$ ) of maternally-reared controls. The hand-rearing also 
largely rescued defective dendrite pruning seen in mother-reared OSN-TeNT animals. Now, the difference between hand-reared control and OSN-TeNT mice was small, if any, at P6 (Fig. 3j). Thus, neuronal activity derived from OSNs plays a minimal role for the normal development of mitral cell dendrites.

\section{Awake neonatal mice show OSN-independent spontaneous activity in mitral cells}

Our genetic experiments raised the possibility that spontaneous activity in the olfactory bulb is important for dendrite pruning. However, to the best of our knowledge, spontaneous activity in neonatal animals $(<\mathrm{P} 6)$ has never been characterized in the olfactory system. To directly examine whether spontaneous activity exists in mitral cells in vivo, we performed in vivo two-photon $\mathrm{Ca}^{2+}$ imaging of the olfactory bulb in awake newborn mice (Fig. 4a). We used mitral/tufted cellspecific GCaMP3 and GCaMP6f mouse lines (transgenic Pcdh21-Cre;Ai38 and Thy1-GCaMP6f, designated M/T-GCaMP3/6f hereafter) to record neuronal activity in mitral/tufted cell dendrites (glomerular layer) ${ }^{49,53,54}$. In vivo imaging of awake neonatal mice at P4 revealed spontaneous neuronal activity in the glomerular layer of the olfactory bulb (Fig. 4b). Spontaneous activity was not seen in ketamine-anesthetized pups (Supplementary Fig. 3a-e), which was similar to earlier studies in other sensory systems ${ }^{9,10,55}$. We also performed $\mathrm{Ca}^{2+}$ imaging of OSNs using an OSN-specific GCaMP3 mouse line (transgenic OMP-tTA;TRE-GCaMP3, designated OSNGCaMP3 hereafter) at P4. However, we did not observe spontaneous activity in OSN axons in the glomerular layer at this age (Fig. 4c). Furthermore, robust spontaneous activity was observed even after naris occlusion (Fig. 4d) and in OSN-TeNT mice (Supplementary Fig. 3f). Thus, the spontaneous activity in the olfactory bulb can be generated without synaptic inputs from OSNs.

\section{The age-specific patterns of spontaneous activity in vivo}

We performed in vivo $\mathrm{Ca}^{2+}$ imaging of mitral/tufted cells during the dendrite remodeling process, from P1 to P6. The pattern of spontaneous activity changed dramatically during development. The spontaneous activity was synchronized among many glomeruli at P1-2, but became desynchronized afterward (Fig. 4e,f, Supplementary Fig. 4, and Supplementary Video 2,3). We determined the correlation index for each time bin ( 1 frame, 0.429 or $0.564 \mathrm{sec}$ ) based on percentages of co-active glomeruli, which were also normalized by the firing frequency (See Methods). Based on the correlation index, each event was classified into highly correlated (H-) events and more sporadic lowly correlated (L-) events ${ }^{56}$. A high ratio of H-events was observed only at P1-3, and L-events dominated afterward (Fig. 4g). At all stages, the activity was synchronized within each glomerulus as is known in the adult; this is likely due to dense electrical coupling via gap junctions within a glomerulus ${ }^{57,58}$. Spike frequency and amplitude was variable among glomeruli and ages (Supplementary Fig. 4b,c).

We also examined the spatial distribution of correlated events. We determined the correlation between two glomeruli normalized by their individual firing frequency (Spike Timing Tiling Coefficient, see Methods) ${ }^{59}$, and examined the spatial distribution of glomeruli with 
correlated activity. At P1-2, correlated glomeruli were found within 100-150 $\mu \mathrm{m}$, but not at later stages ( $\geq$ P3) (Supplementary Fig. 4d). This again demonstrates two different types of spontaneous activity, synchronized at an early stage and de-synchronized at a later stage.

\section{Spontaneous activity is generated in isolated olfactory bulb in vitro}

To examine whether the spontaneous activity is generated without any inputs from other brain regions, we performed $\mathrm{Ca}^{2+}$ imaging of acute olfactory bulb slices in vitro, thus removing any external inputs. The olfactory bulb was excised from M/T-GCaMP3/6f mice and the dorsal halves of the bulb were continuously perfused with artificial cerebrospinal fluid (ACSF) (Fig. 5a). Similar age-specific patterns of spontaneous activity were observed in this preparation as in vivo, both in the glomerular layer (Fig. 5b,c) and in mitral cell somata (Supplementary Fig. 2a). Notably, the pattern of spontaneous activity was synchronized at an early stage, and desynchronized at a later stage (Fig. 5b,c, Supplementary Video 4,5), as was seen in vivo. Hevents were more frequent at P2 than at P6 (Fig. 5d). We determined cross-correlation values for activity in all glomeruli imaged at P2 and P6 in vitro. A high correlation between glomeruli was found for many pairs at P2, but not at P6 (Fig. 5e).

At P2, the H-events were in fact propagating waves (Fig. 5b, Supplementary Fig. 5a-e,h, Supplementary Video 4) and were broadly, but not strictly, reproducible as seen in the crosscorrelogram (Supplementary Fig. 5a-c). The propagation speed was much slower than those of action potentials (Supplementary Fig. 5e,h), suggesting that it is a multi-synaptic propagation of bursting activity. The spike frequency and amplitude were variable among glomeruli (Supplementary Fig. 6f,g).

\section{NMDARs are required for the dendrite pruning cell-autonomously}

So far, we have excluded the contribution of OSN-derived and centrifugal inputs as the possible origins of the spontaneous activity. Leading to the question, how is spontaneous activity generated and propagated within the olfactory bulb? We investigated the possible origin of spontaneous activity using pharmacological manipulations of the isolated olfactory bulb. Spontaneous activity was completely eliminated by tetrodotoxin (TTX), demonstrating that the $\mathrm{Ca}^{2+}$ signals are indeed generated by action potentials (Fig. 6a). When we applied inhibitors to NMDA- and AMPA-type ionotropic glutamate receptors (APV and CNQX, respectively), both the amplitude and frequency of spontaneous activity were reduced (Fig. 6b). Inhibitors for gap junctions also reduced the frequency of spontaneous activity (Fig. 6c, Supplementary Fig. 6a).

In the developing brain, GABA can also act as an excitatory neurotransmitter for some types of neurons, with high intracellular $\mathrm{Cl}^{-}$levels ${ }^{60}$. However, the application of GABA shuts off the spontaneous activity of mitral cells at P2, suggesting that GABA is inhibitory toward mitral cells (Supplementary Fig. 6b). We also analyzed dendrite morphology in mice deficient for NKCC1, a $\mathrm{Na}^{+}-\mathrm{K}^{+}-2 \mathrm{Cl}^{-}$co-transporter essential for maintaining a high $\mathrm{Cl}^{-}$concentration 
inside cells ${ }^{61}$. We did not see any defects in dendrite pruning in the NKCC1 mutant mouse line (Supplementary Fig. 6c), further excluding the role of excitatory action of GABA in dendrite pruning.

We therefore investigated a role for glutamatergic synaptic transmission. Specifically, we examined a possible role for the NMDA glutamate receptor (NMDAR) in dendrite pruning. We performed a single-cell knockout of Grin1, which encodes an essential subunit of NMDARs, NR1. We electroporated a Cre plasmid into a subset of mitral cells in floxed-Grin1 mice and examined their morphology. We found that dendrite pruning was significantly perturbed in NMDAR-deficient neurons both at P6 and P14 (Fig. 6d,e). At P6, more than half of mitral cells maintained connections to multiple glomeruli. The formation of lateral dendrites was also slightly affected in the NMDAR-deficient neurons (Fig. 6f). Thus, NMDARs regulate the pruning of mitral cell dendrites in a cell-autonomous fashion.

\section{Neurotransmission among mitral/tufted cells is the critical source of activity}

Where is the glutamate coming from? In the isolated olfactory bulb, mitral/tufted cells are the only possible source of glutamatergic neurotransmission. They are known to interact through dendro-dendritic neurotransmission ${ }^{14}$. We therefore tested the possibility that glutamatergic neurotransmission among mitral/tufted cells is required for the dendrite pruning. To block the neurotransmission among mitral/tufted cells, we generated Pcdh21-Cre;R26-CAG-loxP-TeNT (designated M/T-TeNT) mice, in which TeNT is specifically expressed in mitral/tufted cells. Immunoreactivity for VAMP2 was reduced in the external plexiform layer of the olfactory bulb (Fig. 7a). Contrary to the OSN-TeNT mice, M/T-TeNT mice did not show growth defects (data not shown). As we used the transgenic Pcdh21-Cre line, a small fraction of mitral/tufted cells may have failed to express Cre, as has been seen in the adult ${ }^{49}$. Nevertheless, we confirmed a reduction in spontaneous activity by using in vivo $\mathrm{Ca}^{2+}$ imaging of awake M/T-TeNT pups at P6. The frequency of glomerular spikes were significantly reduced, but not eliminated in M/T-TeNT mice (Fig. 7b,c). The glomerulus-specific L events were particularly reduced (Supplementary ig. 7). Morphological analysis revealed that M/T-TeNT mice show a significant reduction in the percentage of mitral cells innervating a single glomerulus, both at P6 and P14 (Fig. 7d). Thus, glutamatergic neurotransmission among mitral/tufted cells is important for generating spontaneous activity, and for the normal dendrite pruning in Phase II.

\section{DISCUSSION}

\section{Step-wise regulation of dendrite remodeling in mitral cells}

While the discrete connectivity of mitral cell dendrites to a single glomerulus has been known since Camillo Golgi's seminal work more than 140 years ago ${ }^{62}$, the mechanisms behind the formation of this highly precise connection has been a long-standing mystery. In the present 
study, we found that spontaneous neuronal activity plays a key role in establishing the precise dendritic connectivity. This is in stark contrast to the strictly genetically-defined wiring of the Drosophila projection neurons ${ }^{16}$. Together with the "one neuron-one receptor" and the "one glomerulus-one receptor" rules for OSNs ${ }^{12}$, this discrete dendrite wiring ensures segregated olfactory information processing, from OSNs, expressing a defined odorant receptor, to distinct sets of mitral/tufted cells innervating a specific glomerulus (Fig. 7e).

The dendrite remodeling process of mitral cells can be divided into two major steps, while they may be partially overlapping in time (Fig. 1g). In the first step (Phase I), mitral cells reduce their exuberant dendritic processes, while strengthening their surviving dendrites by forming ramified tufted structures and excitatory synapses within one or a few glomeruli. This step occurs independently of neuronal activity. Indeed, the overexpression of Kir2.1 had minimal impacts on dendritic morphology at this stage, as well as on the initial formation of tufted structures and excitatory synapses. Thus dendrite remodeling at Phase I is likely controlled by a genetic program and/or physical and chemical interactions with OSN axons. For example, the ablation of OSNs has been known to impair the formation of the apical tuft ${ }^{27,63}$. It has also been reported that OSN-dependent Notch signaling regulates dendritic complexity during late embryonic stages ${ }^{36}$. Thus, OSN axons may serve as a scaffold for subsequent glomerular circuit formation. During Phase II, one dendrite is exclusively strengthened to form a thick tufted dendrite, whereas the supernumerary ones are pruned. Neuronal activity is essential for the fine tuning of the dendritic connectivity at this stage, by selecting one out from a few possible targets. As wild-type neurons do not always form multiple tufted dendrites during development (30.3\% at P3) (Fig. 1f), the elimination of previously established tufted dendrites may occur only in this minor population of neurons. However, the overexpression of Kir2.1 results in formation of multiple primary dendrites in majority of mitral cells (80.7\% connecting to multiple glomeruli at P6). This may indicate that neuronal activity is required for the pruning of supernumerary dendrites that could otherwise become mature primary dendrites at a later stage. Activity may also be important for the maintenance of synapses, because Kir2.1-expressing mitral cells tend to show a poor tufted structure at a later stage (P28, data not shown).

\section{Origins of spontaneous activity}

The origins of spontaneous activity used in sensory circuit formation have been extensively studied in visual, auditory, and somatosensory systems. For example, in the visual system, propagating spontaneous activity (known as retinal waves) generated in the retinal ganglion cells regulates the fine tuning of the retinotopic map as well as eye-specific segregation in lateral geniculate nucleus (LGN) ${ }^{1,4,8}$. In particular, the early postnatal retinal wave (Stage II wave) is driven by acetylcholine derived from starburst amacrine cells. In the auditory system, spontaneous ATP release activates hair cells, and subsequently afferent nerve fibers, contributing to tonotopic map formation ${ }^{5,10,64}$. In the somatosensory system, spontaneous activity produced in the periphery by unknown mechanisms contributes to the barrel map formation in the 
somatosensory cortex ${ }^{55}$. In addition, there is an ongoing debate regarding the roles of spontaneous vs. evoked activity. Nevertheless, the central hypothesis in the field has been that the peripherally-derived neuronal activity instructs higher sensory circuits.

In the current study, however, we found that the spontaneous activity generated within the mitral/tufted cell network establishes the discrete wiring of mitral cell dendrites. Dendrodendritic glutamatergic synaptic transmission and/or glutamate spillover among mitral/tufted cells may be a major source of spontaneous activity that is required for dendrite pruning. Interestingly, the developmental changes of spontaneous activity from a synchronized to desynchronized pattern (Fig. 4, 5) is also known for retinal waves ${ }^{65}$. In the visual system, there are three types of retinal waves with different roles ${ }^{1}$. It would be interesting to test in the future whether the different types of spontaneous activity in the olfactory bulb contributes to different aspects of circuit development in the olfactory and higher circuits ${ }^{66,67}$.

\section{How do mitral cells choose just one glomerulus?}

During the dendrite remodeling of mitral cells, one of the dendrites is strengthened to form strong synaptic connections, whereas the supernumerary ones are pruned. In particular, the pruning of primary dendrites is mediated by neuronal activity. We also found that this process is mediated by NMDARs. NMDARs are known to mediate the coincidence detection of pre- and post-synaptic activity. This mechanism is hypothesized to mediate the Hebbian-based remodeling of neurites, where "cells that fire together wire together" and "out of synch, lose your link" ${ }^{68}$. In the visual system, it has been suggested that the eye-specific patterns of spontaneous activity facilitates the eye-specific segregation of visual circuits ${ }^{69}$. Therefore, it is tempting to speculate that the developmental de-synchronization of the spontaneous activity may facilitate the dendrite pruning of mitral cells. It is an important ongoing challenge in the field to establish whether and how specific patterns of activity instruct the wiring specificity in sensory circuits.

Competition and synapse elimination is a wide-spread phenomenon in the developing brain. For example, it has been known that axons from different neurons compete for one target in the neuromuscular junction and cerebellar climbing fiber-Purkinje synapses. However, the mechanisms for the competition and selective synapse elimination remain elusive ${ }^{70,71}$. In mitral cells, competition occurs within a neuron, selecting one "winner" dendrite and eliminating all the other "losers". Neuronal activity mediates the elimination of the "losers". It will be important to determine which molecules mediate the intracellular competition.

In the visual, auditory, and somatosensory circuits, spontaneous activity generated in the periphery propagates all the way to the higher circuits to form coordinated connections as well as organized columnar structures. In contrast, the olfactory bulb sculpts parallel segregated olfactory connections based on its own intrinsic network activity, rather than the OSN-derived activity. An important lesson from our finding is that the autonomous formation of highly organized circuitry is another important principle for the activity-dependent development in 
sensory systems.

\section{ACKNOWLEDGMENTS}

We thank M. Yokoi (Pcdh21-Cre), I. Imayoshi (R26-CAG-LoxP-TeNT), P. Mombaerts (OMPGFP, OMP-Cre), S. Tonegawa (floxed Grin1, TRE-TeNT), H. Zeng (Ai38), K. Svoboda (Thy1GCaMP6f), C. Ron Yu (OMP-tTA knock-in), G.E. Shull (NKCC1 KO) for mouse strains, D. Clapham (NaChBac), C. Cepko (pCA-LNL-tdTomato), P. Soriano (FLPo) for plasmids, A.S. Lowe for image processing software, I.D. Thompson for the neonatal anesthesia protocol, and T. Miyata for instruction of in utero electroporation at E10. We are also grateful to R. Iwata and MT. Ke for valuable advices on imaging and analysis, and M. Nomura, Y. Sakashita, Y. Taniyama, and E. Yamashita for technical assistance. This work was supported by grants from the PRESTO program of the Japan Science and Technology Agency (JST) (T.I.), the JSPS KAKENHI (23680038, 15H05572, 15K14336, 16K14568, 16H06456, and 17H06261 to T.I., 15K14327 and 17K14944 to S.F., 17K14946 to M.N.L.), Sumitomo Foundation (T.I.), Nakajima Foundation (T.I.), and RIKEN CDB intramural grant (T.I.). Imaging experiments were supported by the RIKEN Kobe Light Microscopy Facility and animal experiments were supported by the Laboratory for Animal Resources and Genetic Engineering at the RIKEN Center for Life Science Technologies.

\section{AUTHOR CONTRIBUTIONS}

S.F. performed in utero electroporation, morphological analysis, hand rearing, and $\mathrm{Ca}^{2+}$ imaging of olfactory bulb slices. M.N.L. performed hand rearing and $\mathrm{Ca}^{2+}$ imaging of olfactory bulb in vivo. R.S. performed in utero electroporation at E10. Y.M. and T.S. established in utero electroporation of mitral cells. R. K. and K.K. generated floxed Cnga2. T.I. supervised the project. S.F., M.N.L. and T.I. wrote the manuscript. S.F. and M.N.L. contributed equally to this work.

\section{REFERENCES}

1. Huberman, A.D., Feller, M.B. \& Chapman, B. Mechanisms underlying development of visual maps and receptive fields. Annual review of neuroscience 31, 479-509 (2008).

2. Luo, L. \& Flanagan, J.G. Development of continuous and discrete neural maps. Neuron 56, 284-300 (2007).

3. Feldheim, D.A. \& O'Leary, D.D.M. Visual Map Development: Bidirectional Signaling, Bifunctional Guidance Molecules, and Competition. Cold Spring Harbor Perspectives in Biology 2 (2010).

4. $\quad$ Feller, M.B., Wellis, D.P., Stellwagen, D., Werblin, F.S. \& Shatz, C.J. Requirement for cholinergic synaptic transmission in the propagation of spontaneous retinal waves. Science 272, 1182-1187 (1996).

5. Tritsch, N.X., Yi, E.Y., Gale, J.E., Glowatzki, E. \& Bergles, D.E. The origin of spontaneous activity in the developing auditory system. Nature 450, 50-+ (2007).

6. Triplett, J.W. et al. Retinal Input Instructs Alignment of Visual Topographic Maps. Cell 139, 175-185 (2009). 
7. Rossi, F.M. et al. Requirement of the nicotinic acetylcholine receptor beta 2 subunit for the anatomical and functional development of the visual system. Proceedings of the National Academy of Sciences of the United States of America 98, 6453-6458 (2001).

8. $\quad$ Meister, M., Wong, R.O.L., Baylor, D.A. \& Shatz, C.J. Synchronous Bursts of Action-Potentials in Ganglion-Cells of the Developing Mammalian Retina. Science 252, 939-943 (1991).

9. Ackman, J.B., Burbridge, T.J. \& Crair, M.C. Retinal waves coordinate patterned activity throughout the developing visual system. Nature 490, 219-+ (2012).

10. Babola, T.A. et al. Homeostatic Control of Spontaneous Activity in the Developing Auditory System. Neuron 99, 511-+ (2018).

11. Buck, L. \& Axel, R. A novel multigene family may encode odorant receptors: a molecular basis for odor recognition. Cell 65, 175-187 (1991).

12. Mori, K. \& Sakano, H. How is the Olfactory Map Formed and Interpreted in the Mammalian Brain? Annu Rev Neurosci (2011).

13. Murthy, V.N. Olfactory Maps in the Brain. Annual Review of Neuroscience, Vol 34 34, 233-258 (2011).

14. Imai, T. Construction of functional neuronal circuitry in the olfactory bulb. Semin Cell Dev Biol 35, 180188 (2014).

15. Wilson, R.I. \& Mainen, Z.F. Early events in olfactory processing. Annu Rev Neurosci 29, 163-201 (2006).

16. Hong, W. \& Luo, L. Genetic control of wiring specificity in the fly olfactory system. Genetics 196, 17-29 (2014).

17. Li, H.J. et al. Classifying Drosophila Olfactory Projection Neuron Subtypes by Single-Cell RNA Sequencing. Cell 171, 1206-+ (2017).

18. Hong, W.Z., Mosca, T.J. \& Luo, L.Q. Teneurins instruct synaptic partner matching in an olfactory map. Nature 484, 201-+ (2012).

19. Zhu, H.T. et al. Dendritic patterning by Dscam and synaptic partner matching in the Drosophila antennal lobe. Nature Neuroscience 9, 349-355 (2006).

20. Belluscio, L., Lodovichi, C., Feinstein, P., Mombaerts, P. \& Katz, L.C. Odorant receptors instruct functional circuitry in the mouse olfactory bulb. Nature 419, 296-300 (2002).

21. Imai, T., Sakano, H. \& Vosshall, L.B. Topographic mapping--the olfactory system. Cold Spring Harbor Perspectives in Biology 2, a001776 (2010).

22. Serizawa, S. et al. A neuronal identity code for the odorant receptor-specific and activity-dependent axon sorting. Cell 127, 1057-1069 (2006).

23. Takeuchi, H. et al. Sequential Arrival and Graded Secretion of Sema3F by Olfactory Neuron Axons Specify Map Topography at the Bulb. Cell 141, 1056-1067 (2010).

24. Miyamichi, K., Serizawa, S., Kimura, H.M. \& Sakano, H. Continuous and overlapping expression domains of odorant receptor genes in the olfactory epithelium determine the dorsal/ventral positioning of glomeruli in the olfactory bulb. Journal of Neuroscience 25, 3586-3592 (2005).

25. Imai, T., Suzuki, M. \& Sakano, H. Odorant receptor-derived cAMP signals direct axonal targeting. Science 314, 657-661 (2006).

26. Imai, T. et al. Pre-target axon sorting establishes the neural map topography. Science 325, 585-590 (2009).

27. Kobayakawa, K. et al. Innate versus learned odour processing in the mouse olfactory bulb. Nature 450, 503-U505 (2007).

28. Inokuchi, K. et al. Nrp2 is sufficient to instruct circuit formation of mitral-cells to mediate odour-induced attractive social responses. Nature Communications 8 (2017). 
29. Imamura, F., Ayoub, A.E., Rakic, P. \& Greer, C.A. Timing of neurogenesis is a determinant of olfactory circuitry. Nature Neuroscience 14, 331-337 (2011).

30. Malun, D. \& Brunjes, P.C. Development of olfactory glomeruli: Temporal and spatial interactions between olfactory receptor axons and mitral cells in opossums and rats. Journal of Comparative Neurology 368, 116 (1996).

31. Katz, L.C. \& Shatz, C.J. Synaptic activity and the construction of cortical circuits. Science 274, 1133-1138 (1996).

32. Lin, D.M. et al. Formation of precise connections in the olfactory bulb occurs in the absence of odorantevoked neuronal activity. Neuron 26, 69-80 (2000).

33. Ma, L.M. et al. A Developmental Switch of Axon Targeting in the Continuously Regenerating Mouse Olfactory System. Science 344, 194-197 (2014).

34. Nishizumi, H. et al. Primary dendrites of mitral cells synapse unto neighboring glomeruli independent of their odorant receptor identity. Communications biology 2, 14 (2019).

35. Imamura, F. \& Greer, C.A. Pax6 regulates Tbr1 and Tbr2 expressions in olfactory bulb mitral cells. Molecular and Cellular Neuroscience 54, 58-70 (2013).

36. Muroyama, Y., Baba, A., Kitagawa, M. \& Saito, T. Olfactory Sensory Neurons Control Dendritic Complexity of Mitral Cells via Notch Signaling. Plos Genet 12 (2016).

37. Saito, T. In vivo electroporation in the embryonic mouse central nervous system. Nat Protoc 1, 1552-1558 (2006).

38. Blanchart, A., De Carlos, J.A. \& Lopez-Mascaraque, L. Time frame of mitral cell development in the mice olfactory bulb. Journal of Comparative Neurology 496, 529-543 (2006).

39. Ke, M.T., Fujimoto, S. \& Imai, T. SeeDB: a simple and morphology-preserving optical clearing agent for neuronal circuit reconstruction. Nat Neurosci 16, 1154-1161 (2013).

40. Ke, M.T. et al. Super-Resolution Mapping of Neuronal Circuitry With an Index-Optimized Clearing Agent. Cell Rep 14, in press (2016).

41. Matsutani, S. \& Yamamoto, N. Differentiation of mitral cell dendrites in the developing main olfactory bulbs of normal and naris-occluded rats. Journal of Comparative Neurology 418, 402-410 (2000).

42. Arnold, D.B. \& Clapham, D.E. Molecular determinants for subcellular localization of PSD-95 with an interacting K+ channel. Neuron 23, 149-157 (1999).

43. Hayashi-Takagi, A. et al. Labelling and optical erasure of synaptic memory traces in the motor cortex. Nature 525, 333-+ (2015).

44. Wong, R.O.L. \& Ghosh, A. Activity-dependent regulation of dendritic growth and patterning. Nature Reviews Neuroscience 3, 803-812 (2002).

45. Burrone, J., O'Byrne, M. \& Murthy, V.N. Multiple forms of synaptic plasticity triggered by selective suppression of activity in individual neurons. Nature 420, 414-418 (2002).

46. Ren, D.J. et al. A prokaryotic voltage-gated sodium channel. Science 294, 2372-2375 (2001).

47. Bando, Y. et al. Control of Spontaneous Ca2+ Transients Is Critical for Neuronal Maturation in the Developing Neocortex. Cereb Cortex 26, 106-117 (2016).

48. Grosmaitre, X., Santarelli, L.C., Tan, J., Luo, M. \& Ma, M. Dual functions of mammalian olfactory sensory neurons as odor detectors and mechanical sensors. Nature neuroscience 10, 348-354 (2007).

49. Iwata, R., Kiyonari, H. \& Imai, T. Mechanosensory-Based Phase Coding of Odor Identity in the Olfactory Bulb. Neuron 96, 1139-+ (2017).

50. Connelly, T., Savigner, A. \& Ma, M.H. Spontaneous and sensory-evoked activity in mouse olfactory sensory neurons with defined odorant receptors. J Neurophysiol 110, 55-62 (2013). 
51. Yamamoto, M. et al. Reversible suppression of glutamatergic neurotransmission of cerebellar granule cells in vivo by genetically manipulated expression of tetanus neurotoxin light chain. Journal of Neuroscience 23, 6759-6767 (2003).

52. Yu, C. et al. Spontaneous neural activity is required for the establishment and maintenance of the olfactory sensory map. Neuron 42, 553-566 (2004).

53. Dana, H. et al. Thy1-GCaMP6 Transgenic Mice for Neuronal Population In Vivo. PLoS One 9 (2014).

54. Zariwala, H.A. et al. A Cre-Dependent GCaMP3 Reporter Mouse for Neuronal Imaging In Vivo. Journal of Neuroscience 32, 3131-3141 (2012).

55. Mizuno, H. et al. Patchwork-Type Spontaneous Activity in Neonatal Barrel Cortex Layer 4 Transmitted via Thalamocortical Projections. Cell Rep 22, 123-135 (2018).

56. Siegel, F., Heimel, J.A., Peters, J. \& Lohmann, C. Peripheral and Central Inputs Shape Network Dynamics in the Developing Visual Cortex In Vivo. Current Biology 22, 253-258 (2012).

57. Schoppa, N.E. \& Westbrook, G.L. Glomerulus-specific synchronization of mitral cells in the olfactory bulb. Neuron 31, 639-651 (2001).

58. Maher, B.J., McGinley, M.J. \& Westbrook, G.L. Experience-dependent maturation of the glomerular microcircuit. Proceedings of the National Academy of Sciences of the United States of America 106, 1686516870 (2009).

59. Cutts, C.S. \& Eglen, S.J. Detecting Pairwise Correlations in Spike Trains: An Objective Comparison of Methods and Application to the Study of Retinal Waves. Journal of Neuroscience 34, 14288-14303 (2014).

60. Ben-Ari, Y. Excitatory actions of GABA during development: The nature of the nurture. Nature Reviews Neuroscience 3, 728-739 (2002).

61. Wang, D.D. \& Kriegstein, A.R. GABA regulates excitatory synapse formation in the neocortex via NMDA receptor activation. Journal of Neuroscience 28, 5547-5558 (2008).

62. Shepherd, G.M., Greer, C.A., Mazzarello, P. \& Sassoe-Pognetto, M. The first images of nerve cells: Golgi on the olfactory bulb 1875. Brain Research Reviews 66, 92-105 (2011).

63. Leo, J.M.C. \& Brunjes, P.C. Neonatal focal denervation of the rat olfactory bulb alters cell structure and survival: a Golgi, Nissl and confocal study. Dev Brain Res 140, 277-286 (2003).

64. Wang, H.C. et al. Spontaneous Activity of Cochlear Hair Cells Triggered by Fluid Secretion Mechanism in Adjacent Support Cells. Cell 163 (2015).

65. Wong, R.O.L., Meister, M. \& Shatz, C.J. Transient Period of Correlated Bursting Activity during Development of the Mammalian Retina. Neuron 11, 923-938 (1993).

66. de Frutos, C.A. et al. Reallocation of Olfactory Cajal-Retzius Cells Shapes Neocortex Architecture. Neuron 92, 435-448 (2016).

67. Gretenkord, S. et al. Coordinated electrical activity in the olfactory bulb gates the oscillatory entrainment of entorhinal networks in neonatal mice. Plos Biology 17 (2019).

68. Shatz, C.J. The Developing Brain. Sci Am 267, 61-67 (1992).

69. Xu, H.P. et al. An Instructive Role for Patterned Spontaneous Retinal Activity in Mouse Visual Map Development. Neuron 70, 1115-1127 (2011).

70. Lichtman, J.W. \& Colman, H. Synapse elimination and indelible memory. Neuron 25, 269-278 (2000).

71. Watanabe, M. \& Kano, M. Climbing fiber synapse elimination in cerebellar Purkinje cells. European Journal of Neuroscience 34, 1697-1710 (2011).

72. Nagai, Y., Sano, H. \& Yokoi, M. Transgenic expression of Cre recombinase in mitral/tufted cells of the olfactory bulb. Genesis 43, 12-16 (2005). 
73. Sakamoto, M. et al. Continuous Postnatal Neurogenesis Contributes to Formation of the Olfactory Bulb Neural Circuits and Flexible Olfactory Associative Learning. Journal of Neuroscience 34, 5788-5799 (2014).

74. Matsuo, T. et al. Genetic dissection of pheromone processing reveals main olfactory system-mediated social behaviors in mice. Proceedings of the National Academy of Sciences of the United States of America 112, E311-E320 (2015).

75. Tsien, J.Z., Huerta, P.T. \& Tonegawa, S. The essential role of hippocampal CA1 NMDA receptordependent synaptic plasticity in spatial memory. Cell 87, 1327-1338 (1996).

76. Potter, S.M. et al. Structure and emergence of specific olfactory glomeruli in the mouse. Journal of Neuroscience 21, 9713-9723 (2001).

77. Nakashiba, T., Young, J.Z., McHugh, T.J., Buhl, D.L. \& Tonegawa, S. Transgenic inhibition of synaptic transmission reveals role of CA3 output in hippocampal learning. Science 319, 1260-1264 (2008).

78. Li, J.S., Ishii, T., Feinstein, P. \& Mombaerts, P. Odorant receptor gene choice is reset by nuclear transfer from mouse olfactory sensory neurons. Nature 428, 393-399 (2004).

79. Flagella, M. et al. Mice lacking the basolateral Na-K-2Cl cotransporter have impaired epithelial chloride secretion and are profoundly deaf. Journal of Biological Chemistry 274, 26946-26955 (1999).

80. Matsuda, T. \& Cepko, C.L. Controlled expression of transgenes introduced by in vivo electroporation. Proceedings of the National Academy of Sciences of the United States of America 104, 1027-1032 (2007).

81. Okamoto, M. et al. TAG-1-assisted progenitor elongation streamlines nuclear migration to optimize subapical crowding. Nature Neuroscience 16, 1556-1566 (2013).

82. Ke, M.T. \& Imai, T. Optical clearing of fixed brain samples using SeeDB. Curr Protoc Neurosci 66, Unit 2 22 (2014).

83. Lowe, A.S., Nikolaou, N., Hunter, P.R., Thompson, I.D. \& Meyer, M.P. A Systems-Based Dissection of Retinal Inputs to the Zebrafish Tectum Reveals Different Rules for Different Functional Classes during Development. Journal of Neuroscience 33, 13946-13956 (2013). 
a
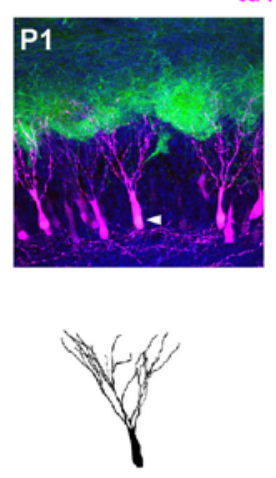

C $\mathrm{P} 1$
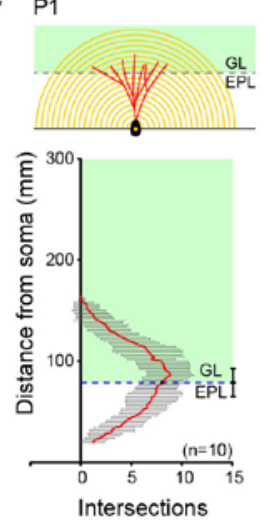

e

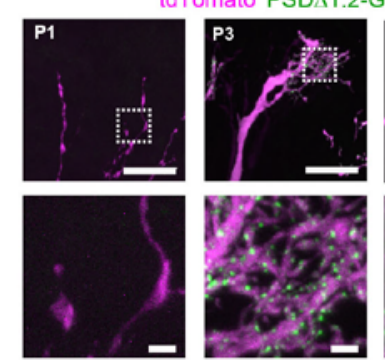

f

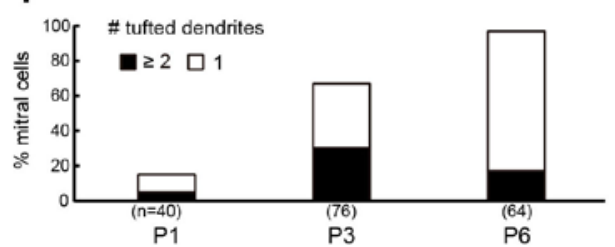

b
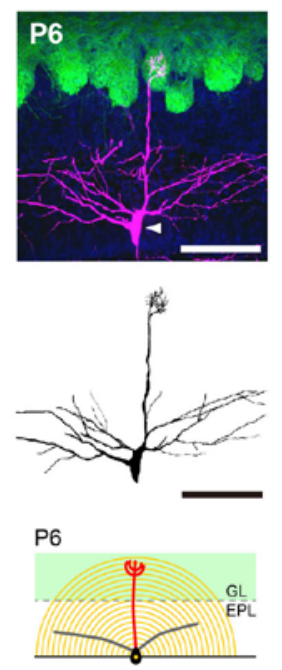

d
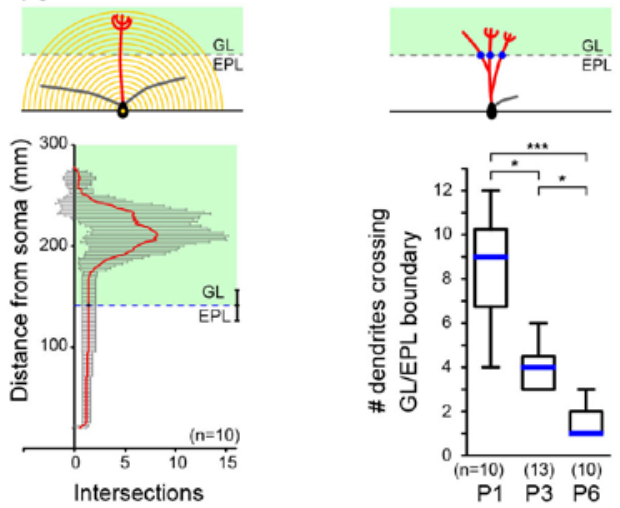

g

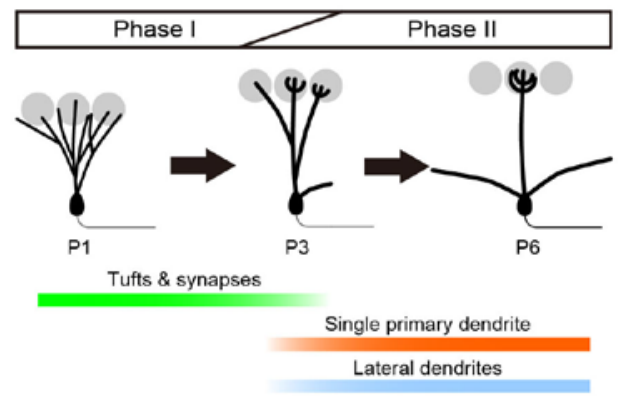

Figure 1 Developmental remodeling of mitral cell dendrites. (a) Representative images of P1, P3, P6 mitral cells labelled with tdTomato (magenta). OMP-GFP knock-in mice were used to label OSN axons (green). Samples were stained with DAPI (blue) and cleared with SeeDB2. Representative neurons (white arrowheads) were reconstructed by Neurolucida and are shown below. (b) In utero electroporation was performed at E12. pCA-LNL-tdTomato and a low concentration of pCAG-Cre were used to label mitral cells sparsely. (c) Modified Sholl analysis of mitral cell dendrites at P1, P3 and P6. Only primary dendrites extending to the glomerular layer (GL) were quantified. Data are the mean \pm SD. EPL, external plexiform layer. (d) The 
number of dendrites extending into the GL at P1, P3 and P6. The number of dendrites crossing GL/EPL boundary was quantified (blue line represents the median, the boxes are the interquartile range, and the whiskers represent the minimum and maximum). ${ }^{*} \mathrm{p}<0.05$, ${ }^{* * *} \mathrm{p}<0.001$ (Kruskal-Wallis test and post hoc Dunn's multiple comparison test). n, number of mitral cells. (e) Postsynaptic structures on primary dendrites at P1, P3 and P6. Primary dendrites (magenta) and PSD $\Delta 1.2-G F P$ puncta (green) are shown. Scale bars, $20 \mu \mathrm{m}$ (top) and $2 \mu \mathrm{m}$ (bottom). (f) Quantitative analysis of the number of dendrites with a tufted structure at P1, P3 and P6. (g) Schematic representation of mitral cell development. Scale bars, $100 \mu \mathrm{m}$. 


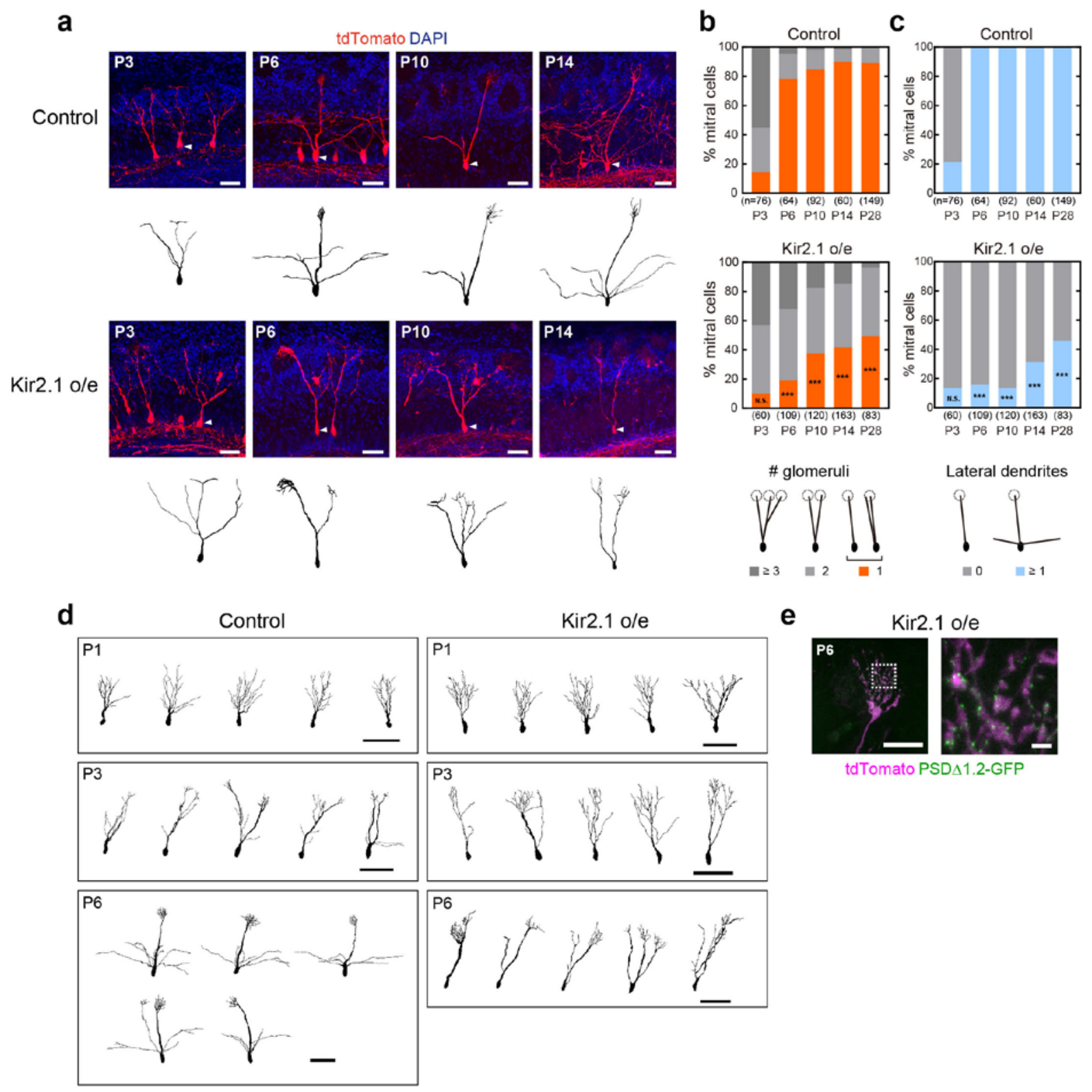

Figure 2 Neuronal activity in mitral cells is essential to form a single primary dendrite. (a) Kir2.1 overexpression in mitral cells perturbs the pruning of mitral cell dendrites and the formation of lateral dendrites when compared to the controls. Representative mitral cell morphologies at P3, P6, P10, and P14 and their reconstructions are shown. Scale bars, $50 \mu \mathrm{m}$. (b) Quantification of primary dendrite pruning in control (top) or Kir2.1-overexperssing (bottom) mitral cells. Percentages of mitral cells with a connection to single (1), double (2), or multiple $(\geq$ 3) glomeruli at P3, P6, P10, P14, and P28 were analyzed. We did not distinguish between tufted vs. non-tufted dendrites in this quantification. N.S., non-significant, ${ }^{* * *} \mathrm{p}<0.001\left(\chi^{2}\right.$ test vs control). n, number of mitral cells. (c) Quantification of lateral dendrite formation in control (top) or Kir2.1-overexperssing (bottom) mitral cells. The percentages of mitral cells with/without lateral dendrites at P3, P6, P10, P14, and P28 are shown. N.S., non-significant, $* * * \mathrm{p}<0.001\left(\chi^{2}\right.$ test vs control). (d) Representative control and Kir2.1-overexpressing mitral cells at P1, P3 and 
P6 reconstructed by Neurolucida. Scale bars, $100 \mu \mathrm{m}$. (e) Postsynaptic structures on the primary dendrites in Kir2.1-overexpressing mitral cells at P6. Primary dendrites (magenta) and PSD $\Delta 1.2-$ GFP puncta (green) are shown. Scale bars, $20 \mu \mathrm{m}$ (left) and $2 \mu \mathrm{m}$ (right). 
a
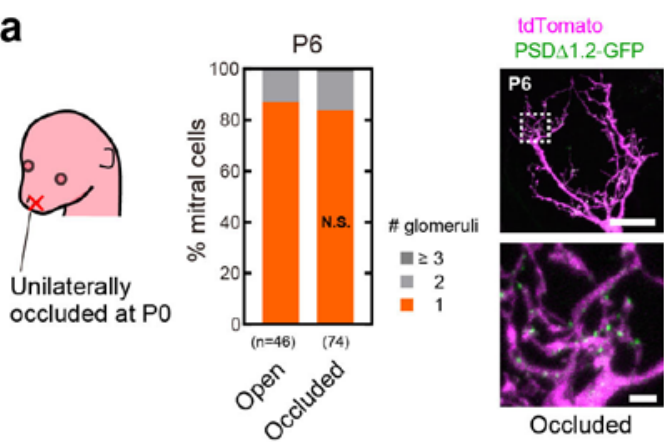

d

g

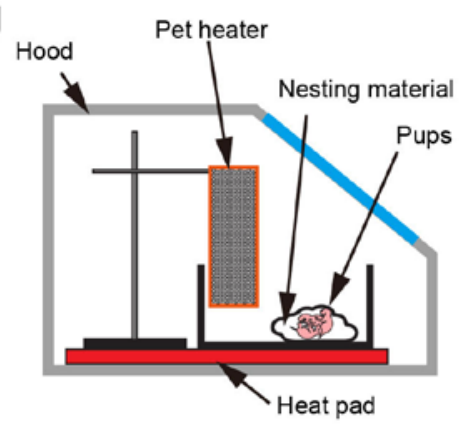

i

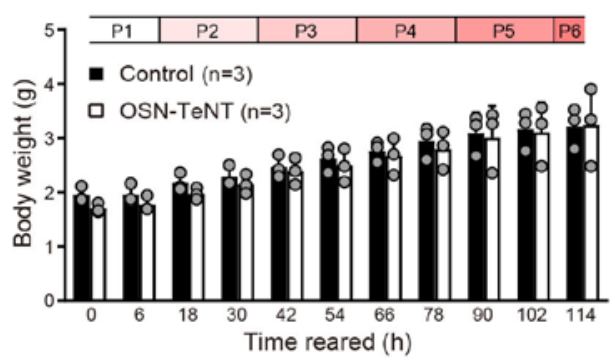

e h b

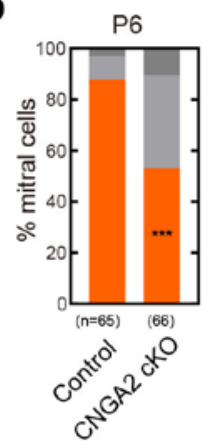

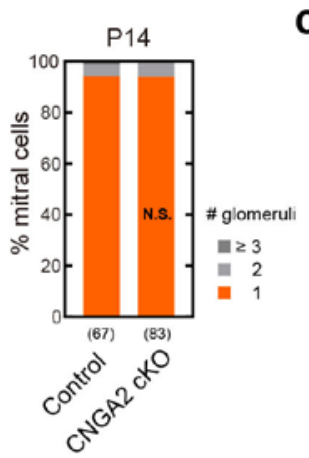

C

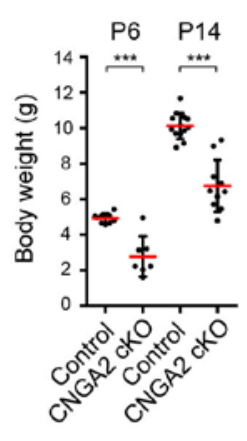

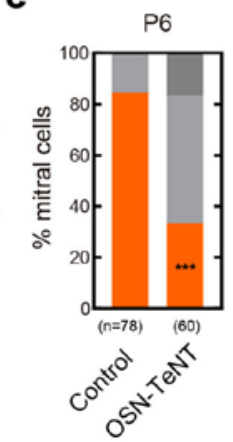

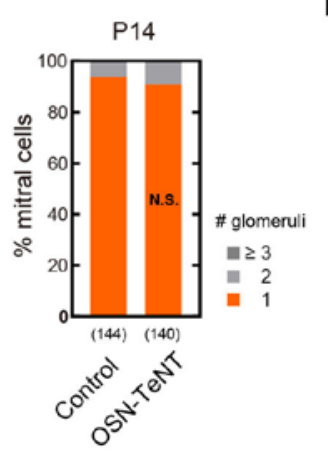

f
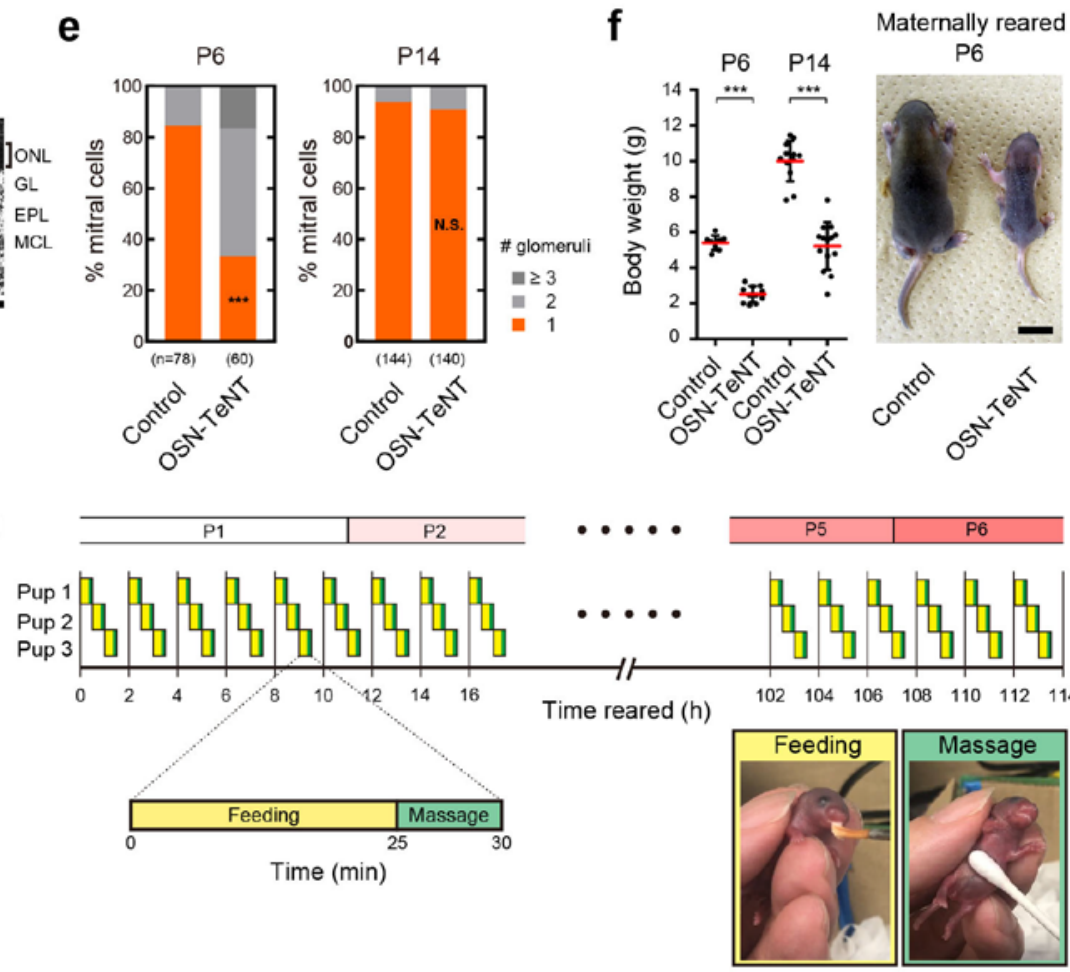

j
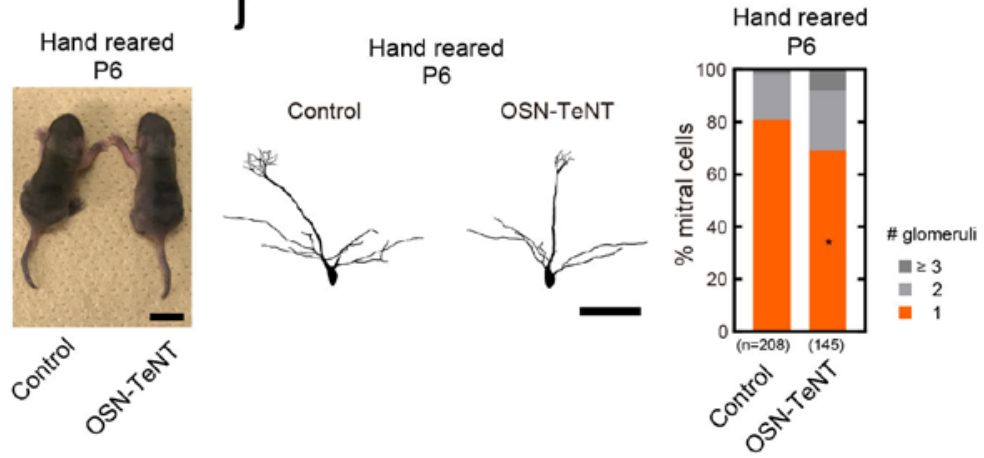

Figure 3 Neuronal activity derived from OSNs is dispensable for dendrite pruning. (a) Quantification of dendrite pruning under unilateral naris occlusion. Naris occlusion did not affect dendrite pruning. N.S., non-significant ( $\chi^{2}$ test occluded vs open). Postsynaptic structures in naris-occluded mice are shown on the right. Scale bars, $20 \mu \mathrm{m}$ (top) and $2 \mu \mathrm{m}$ (bottom). (b) Quantification of dendrite pruning in OSN-specific CNGA2 mutant mice (OMP-Cre;Cnga2 ${ }^{f l / o}$ male). Dendrite pruning was delayed at P6 but recovered at P14, ${ }^{* * *} \mathrm{p}<0.001$, N.S., non- 
significant ( $\chi^{2}$ test vs control). (c) Body weight changes in control and CNGA2 cKO mice at P6 and P14 . *** $<<0.001$ (Welch's t-test). (d) OSN-TeNT mice (OMP-Cre;R26-CAG-LoxP-TeNT). VAMP2 immunostaining of the olfactory bulb section shows an almost complete depletion of VAMP2 signals in the olfactory nerve layer (ONL). GL, glomerular layer; EPL, external plexiform layer; MCL, mitral cell layer. Scale bars, $100 \mu \mathrm{m}$. (e) Quantification of dendrite pruning in OSN-TeNT mice. Dendrite pruning was delayed at P6, but recovered at P14. ${ }^{* * *} \mathrm{p}<0.001$ ( $\chi^{2}$ test vs control). N.S., non-significant, ( $\chi^{2}$ test vs control). (f) Quantifications of body weight in control and OSN-TeNT mice displaying significant weight differences between the two groups. ${ }^{* * *} \mathrm{p}<0.001$ (Welch's t-test, left panel). Representative images of maternally reared control and OSN-TeNT neonates are shown (right). (g) Schematic showing the equipment for the hand-rearing of pups. (h) Up to three pups were hand-reared simultaneously. Mice were fed every two hours with $100 \mu \mathrm{L}$ of milk per gram of body weight each time (yellow boxes). To aid digestion and to prevent bloating, pups were also massaged just after feeding (green boxes). (i) Weight gain during hand rearing. Representative images of control and OSN-TeNT pups are shown on the right. (j) Quantitative analysis of dendrite pruning in hand reared control and OSNTeNT mice. ${ }^{*} \mathrm{p}<0.05\left(\chi^{2}\right.$ test vs control). 

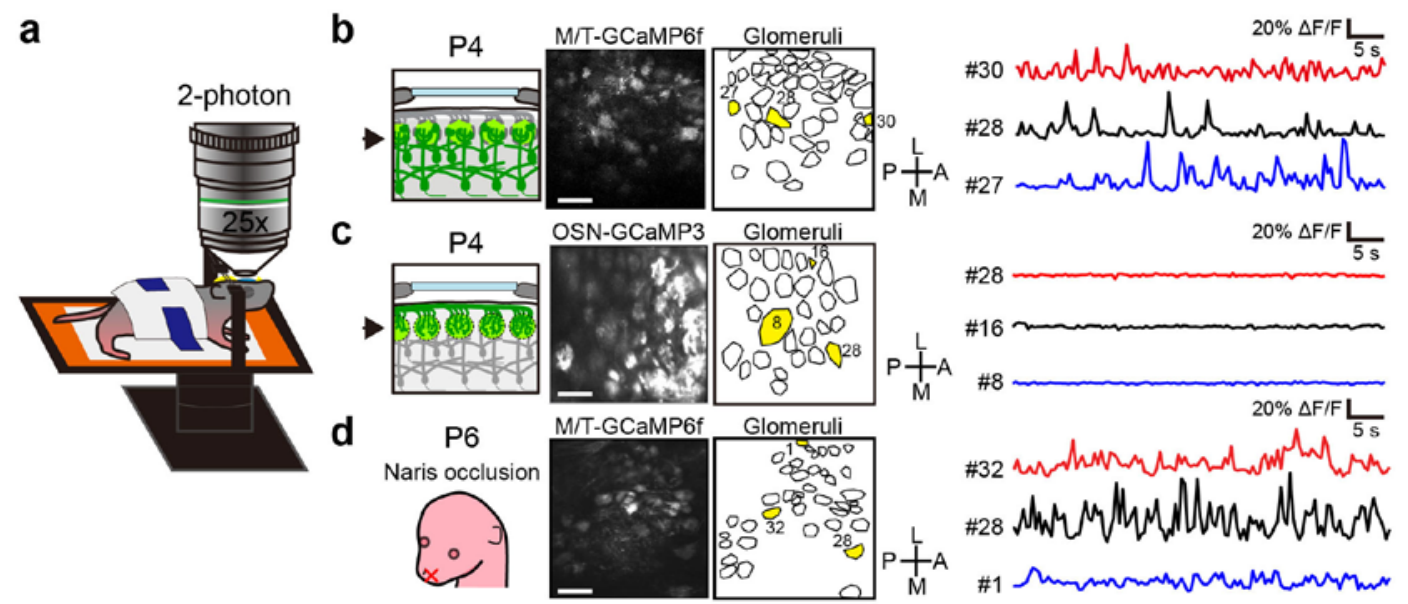

e

P2
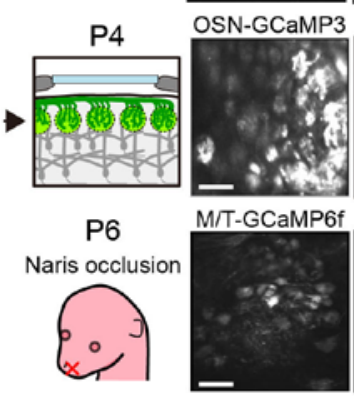

f
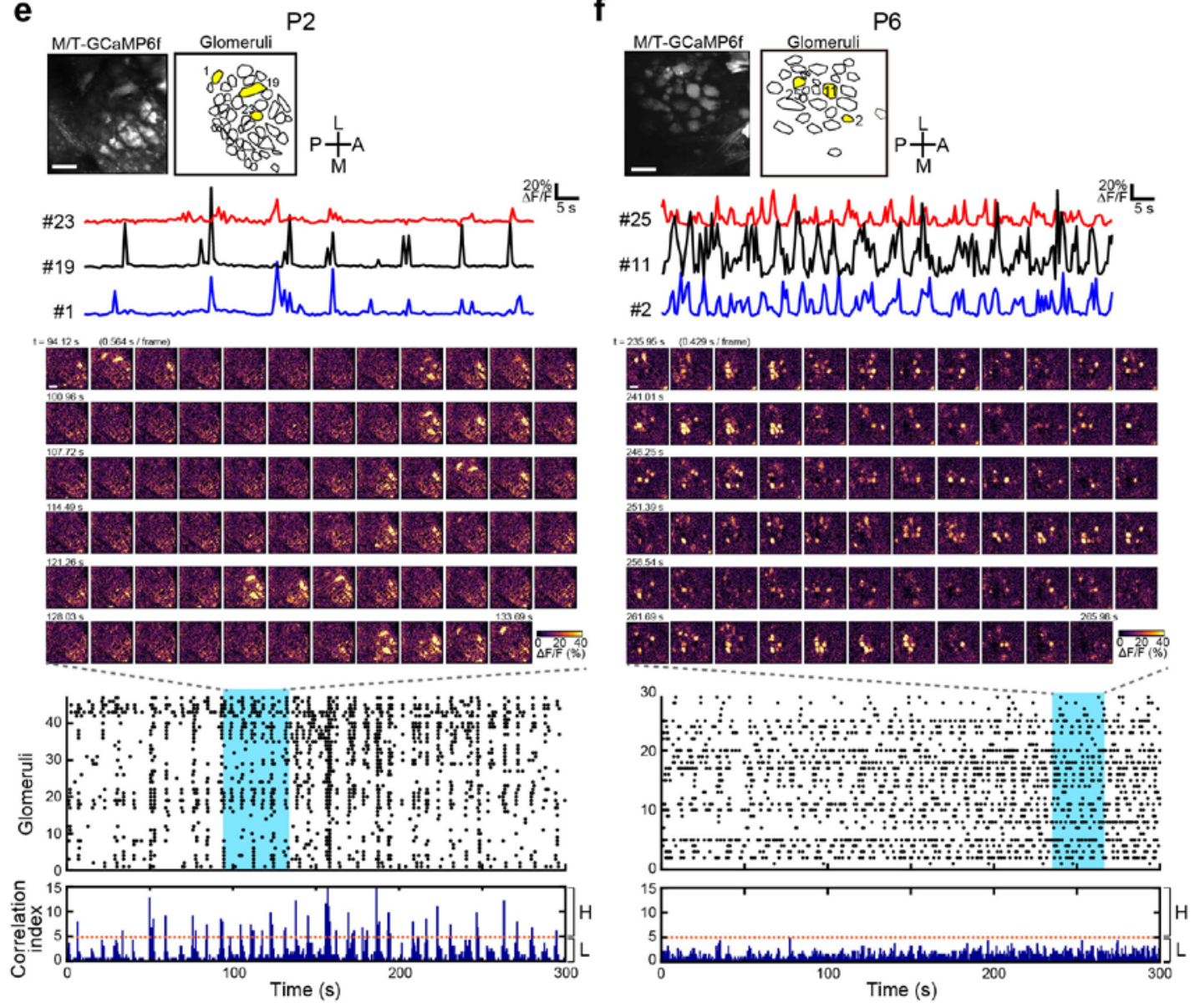

g
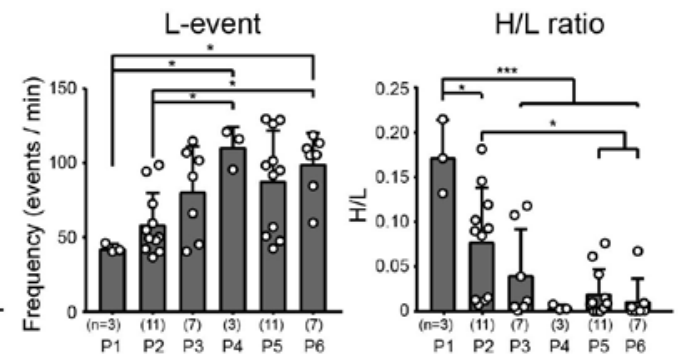
Figure 4 In vivo awake imaging of spontaneous activity in the olfactory bulb. (a) Schematic representation of the imaging set up. A cranial window was made on the left olfactory bulb, and dental cement was used to attach a metal bar for head fixing. (b) GCaMP6f was specifically expressed in M/T-GCaMP6f cells (left). Representative traces are shown (right) for three ROIs (middle, highlighted in yellow), displaying the spontaneous activity. We obtained similar results with M/T-GCaMP3 (data not shown). (c) OSN-GCaMP3 did not show any spontaneous activity in any glomeruli. (d) Naris occlusion from P0-P6 does not affect the spontaneous activity occurring in mitral cells. (e, f) Representative P2 (e) and P6 (f) imaging sessions (top), with individual traces shown below (yellow ROIs in the ROI overlay). A montage of serial $\Delta F / F$ images (middle, highlighted in blue area in the raster plot), raster plots, and correlation index histograms (bottom, See Methods) are also shown. A correlation index threshold of 5 (orange dotted lines) was selected to differentiate between highly correlated $(\mathrm{H}-)$ and lowly correlated (L-) events. A, anterior; P, posterior; M, medial; L, lateral. See also Supplementary Video 2, 3. (g) Developmental changes in H-event frequency, L-event frequency, and the ratio of $\mathrm{H}$ to $\mathrm{L}$ events, respectively. One-way ANOVA with Tukey post hoc tests, ${ }^{*} \mathrm{p}<0.05,{ }^{* *} \mathrm{p}<0.01$, $* * * \mathrm{p}<0.001$, error bars represent SD. n, number of imaging sessions from 2, 4, 3, 3, 3, and 3 animals with total imaging times of 50.01, 211.46, 127.07, 57.87, 235.13, and 126.48 minutes for P1-P6, respectively. More detailed data for P1-P6 data are in Supplementary Figure 4. Scale bars, $100 \mu \mathrm{m}$. 
a

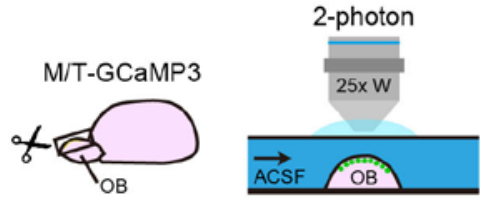

b

P2

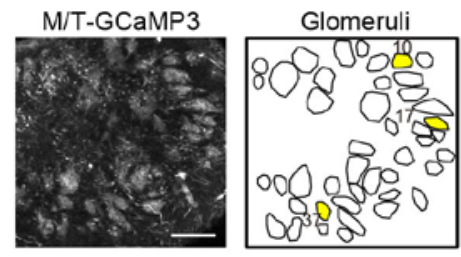

C

P6

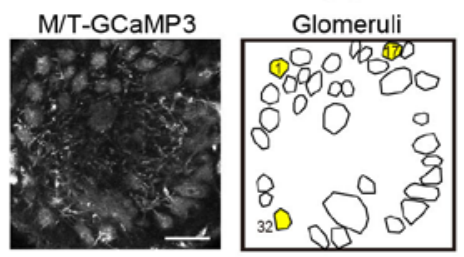

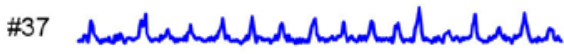

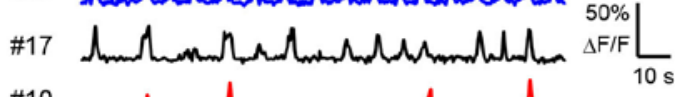

\#10

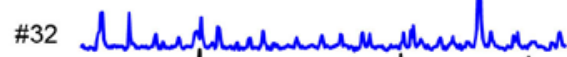

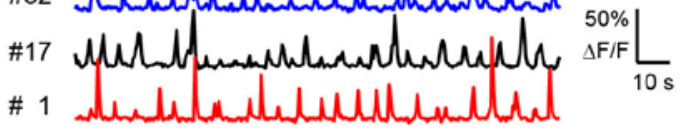
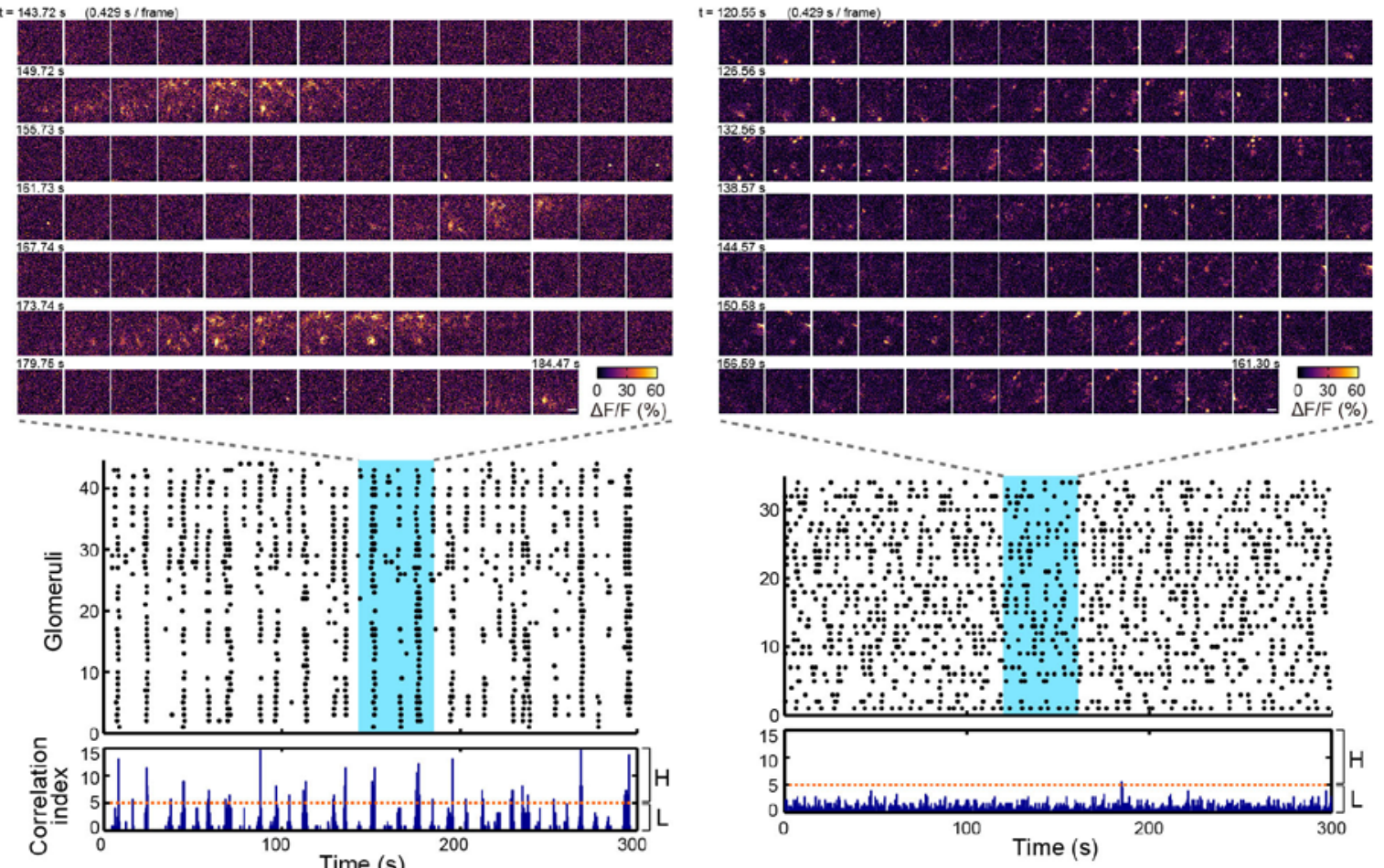

d
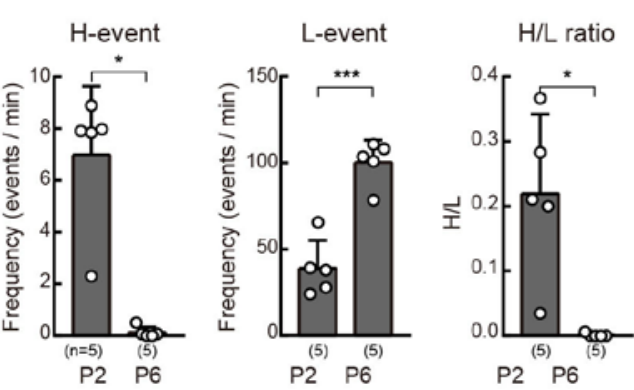

e

P2
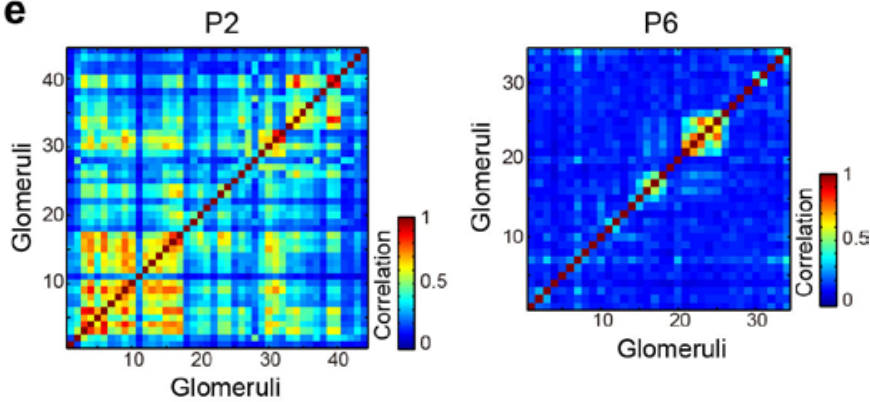

Figure 5 Spontaneous activity in the isolated olfactory bulb in vitro. (a) Schematic of the slice imaging set up. Isolated olfactory bulb (OB) was continuously perfused with artificial cerebrospinal fluid (ACSF). (b, c) Olfactory bulb slices of M/T-GCaMP3 mice were imaged at P2 (b) and P6 (c). Representative imaging sessions (top), with individual traces shown below 
(yellow ROIs in the ROI overlay). A montage of serial $\Delta \mathrm{F} / \mathrm{F}$ images (middle, highlighted in blue area in the raster plot), raster plots, and correlation index histograms (bottom) are also shown. A correlation index threshold of 5 (orange dotted lines) was selected to differentiate between highly correlated (H-) and lowly correlated (L-) events. We obtained similar results with M/T-

GCaMP6f. See also Supplementary Videos 4, 5. (d) Developmental changes of H- and L-events in olfactory bulb slices. Data are mean \pm SD. $n=5$ (P2), 5 (P6) sessions from 3 animals each. ${ }^{*} \mathrm{p}<0.05,{ }^{* *} \mathrm{p}<0.01$ (Welch's t test). (e) Cross-correlation matrix for P2 and P6 data. Scale bars, $100 \mu \mathrm{m}$. 
a

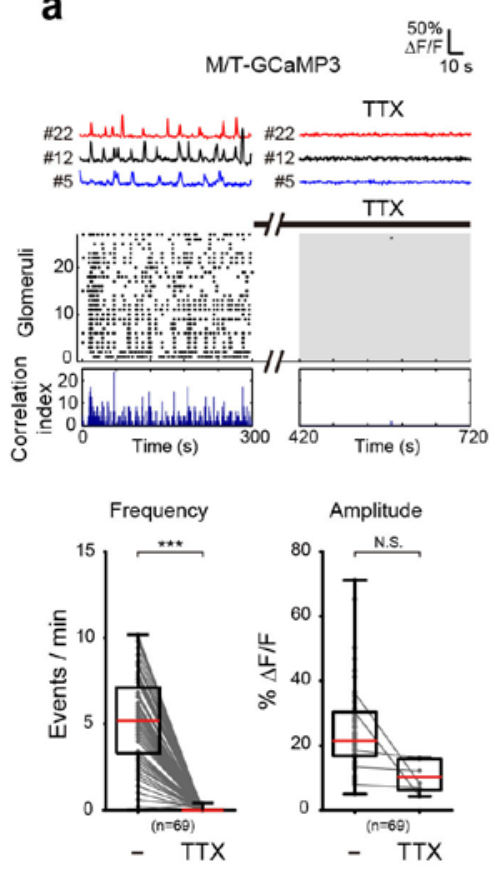

b
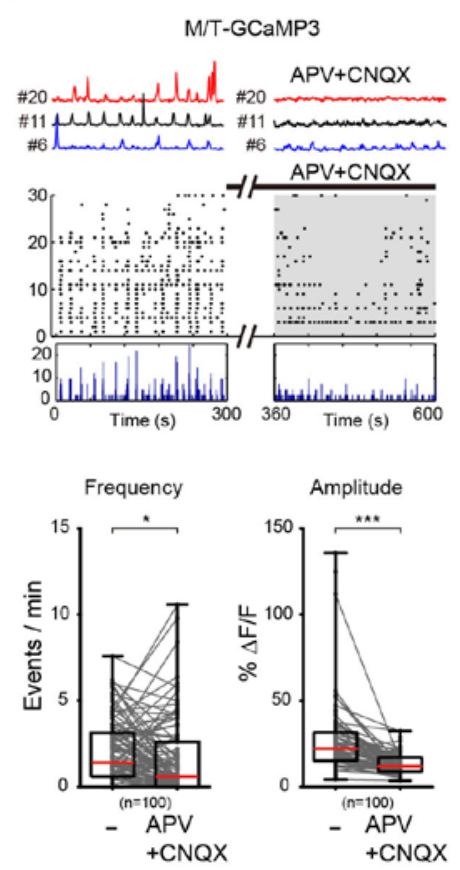

C

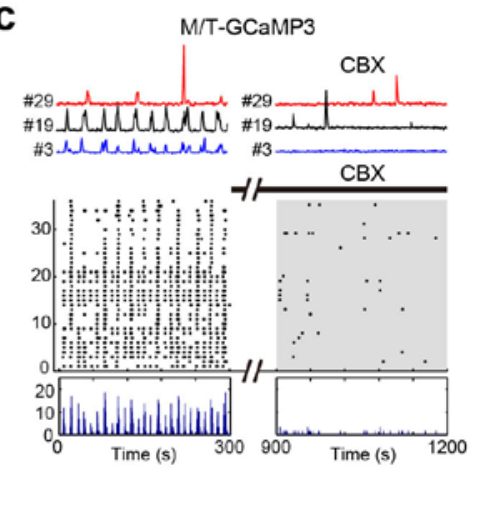

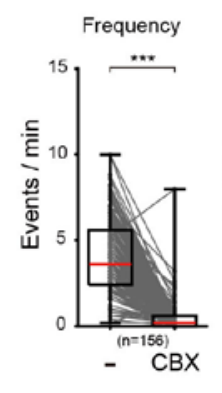

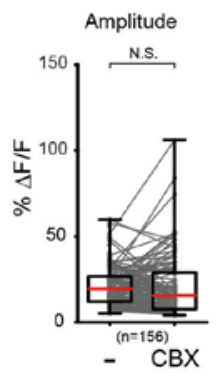

d
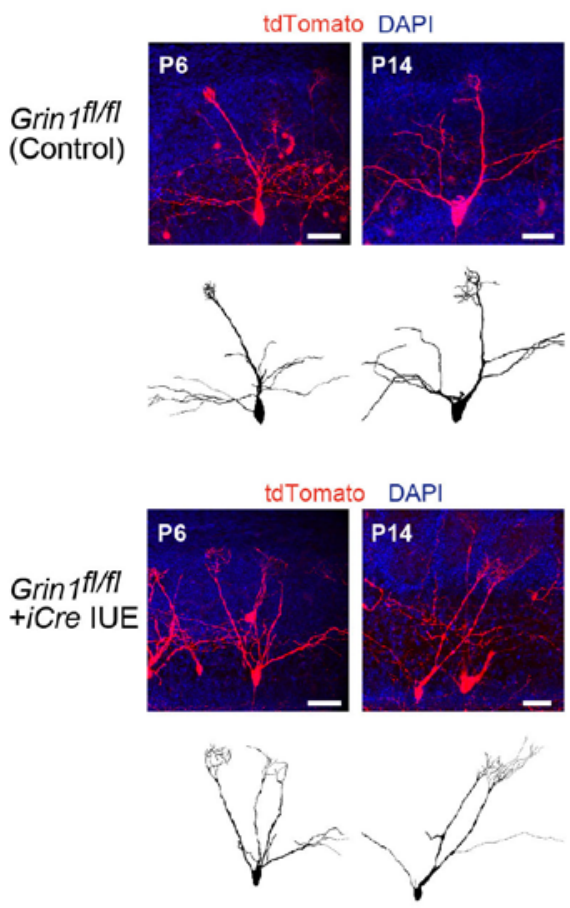

e
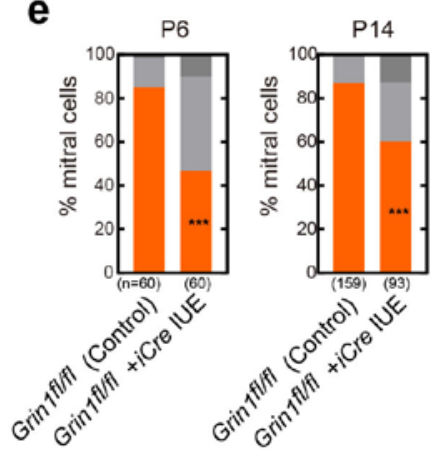

f
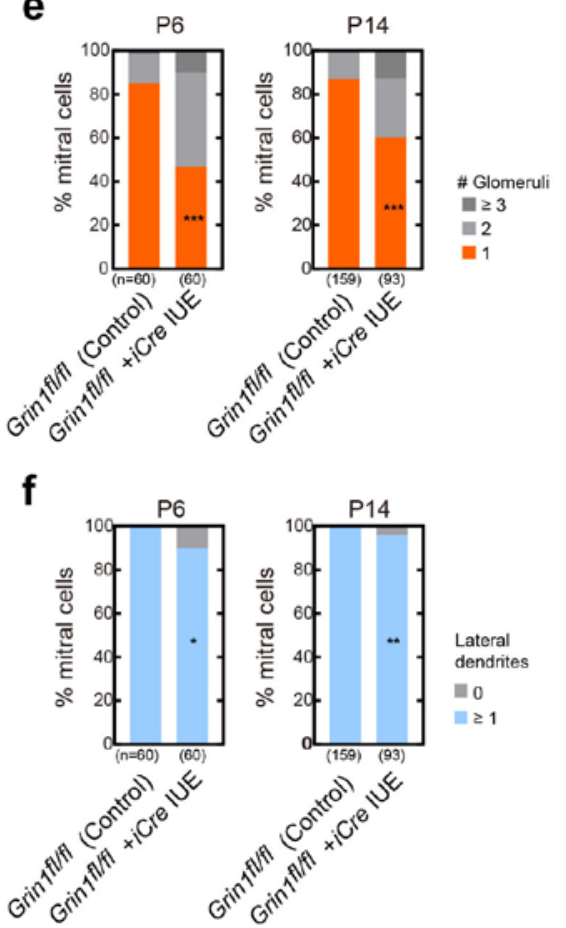

Figure 6 Glutamatergic neurotransmission is required for spontaneous activity and establishing a single primary dendrite. (a) Voltage-gated sodium channels are required for spontaneous activity. Both the frequency and amplitude of glomerular spikes were reduced when TTX was added to P2 olfactory bulb slices. $\mathrm{n}=69$ glomeruli from 3 animals. N.S., non-significant, $*_{* *} \mathrm{p}<0.001$ (Wilcoxon signed-rank test). (b) Application of APV and CNQX shows that ionotropic glutamate receptors are required for spontaneous activity at P2-3 olfactory bulb slices. $\mathrm{n}=100$ glomeruli from 4 animals. ${ }^{*} \mathrm{p}<0.05,{ }^{* * *} \mathrm{p}<0.001$ (Wilcoxon signed-rank test). (c) 
Application of Carbenoxolone (CBX) shows that gap junctions are also required for spontaneous activity at P2-3 olfactory bulb slices. $\mathrm{n}=156$ glomeruli from 4 animals. N.S., non-significant, $* * * \mathrm{p}<0.001$ (Wilcoxon signed-rank test). (d) Representative dendritic morphology of wild-type and NR1 (Grin1)-deficient mitral cells. Scale bars, $50 \mu \mathrm{m}$. (e) Quantification of primary dendrite pruning in NR1 single-cell knockout. Even at P14, a significant fraction of mitral cells ( 40\%) retained connections to multiple glomeruli. ${ }^{* * *} \mathrm{p}<0.001\left(\chi^{2}\right.$-test vs control). (f) Quantification of lateral dendrite formation. Lateral dendrite formation was only slightly affected by the NR1 single-cell knockout. ${ }^{*} \mathrm{p}<0.05,{ }^{* *} \mathrm{p}<0.01$ ( $\chi^{2}$-test vs control) 
a

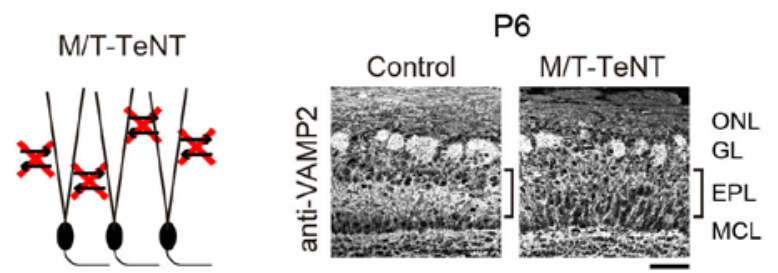

b

In vivo imaging

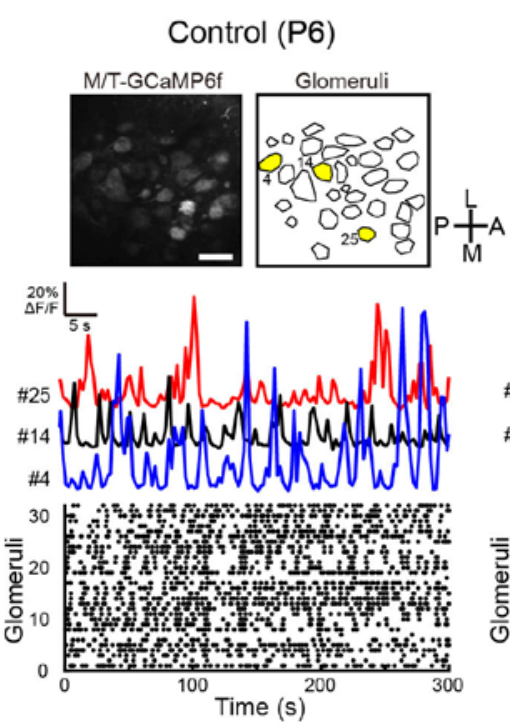

$\mathrm{M} / \mathrm{T}-\mathrm{TeNT}(\mathrm{P} 6)$

M/T-GCaMP6f
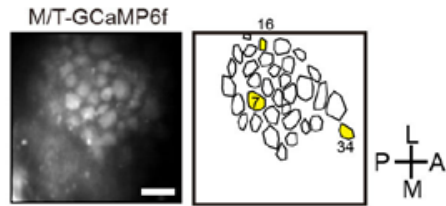

C
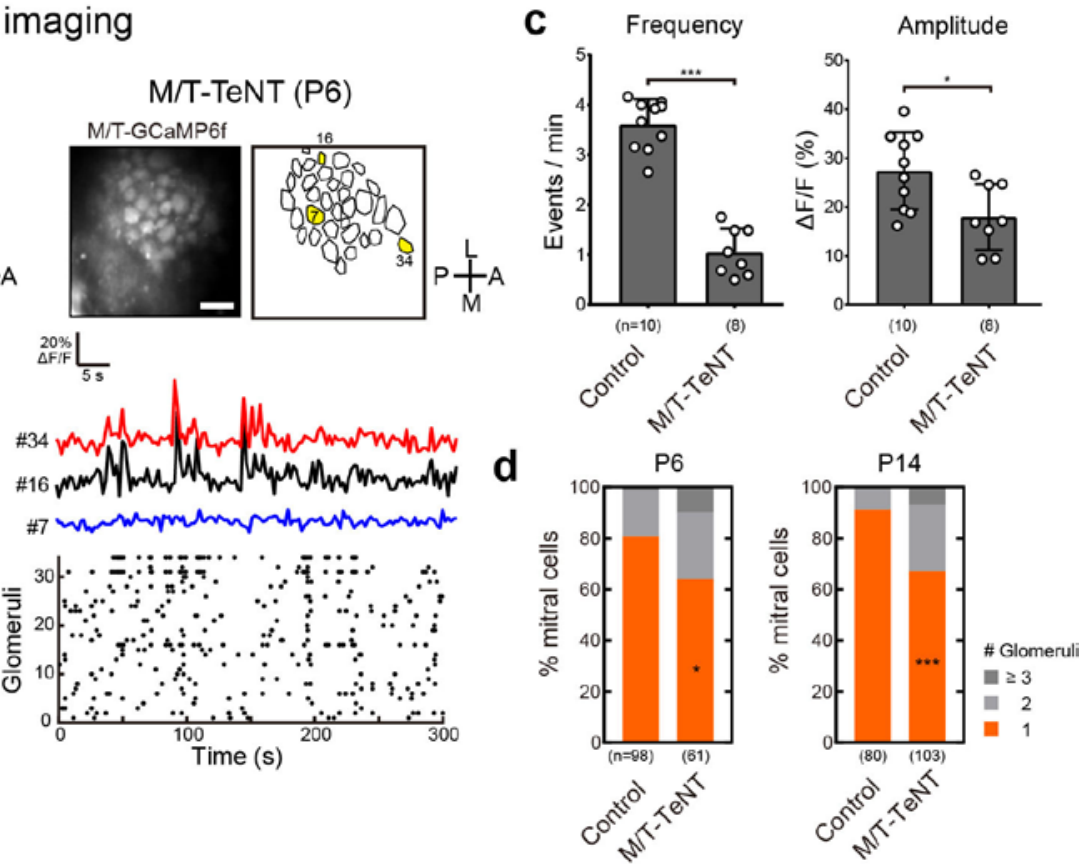

e

Immature

Mature

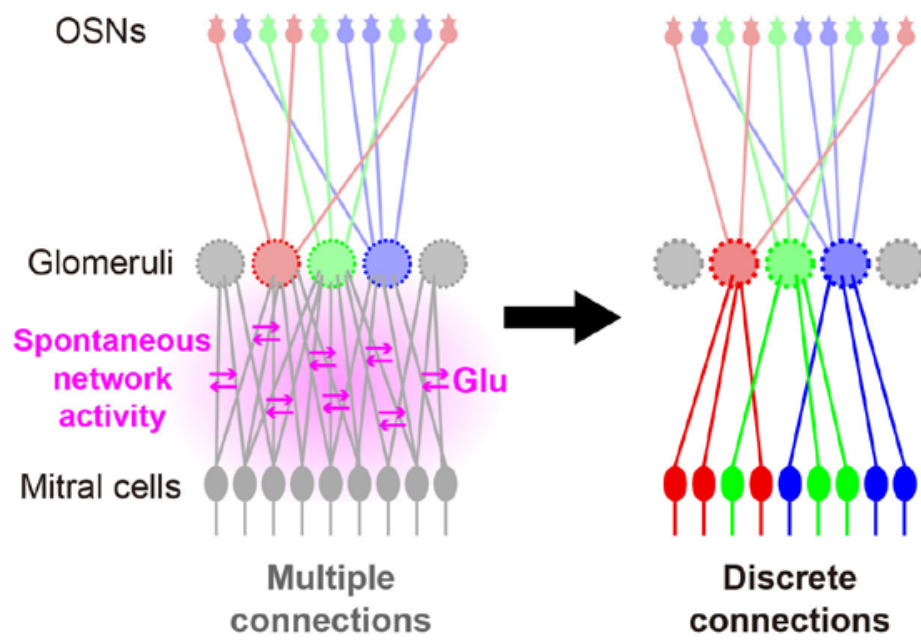

Figure 7 Spontaneous network activity among mitral/tufted cells is required for establishing a single primary dendrite. (a) M/T-TeNT mice (Pcdh21-Cre;R26-CAG-LoxP-TeNT). VAMP2 immunostaining was reduced in M/T-TeNT mice, particularly in the external plexiform layer of the olfactory bulb. Scale bars, $100 \mu \mathrm{m}$. (b) Crossing these mice with the MT-GCaMP6f allowed us to visualize neuronal activity in M/T-TeNT mice (P6). Representative $\mathrm{Ca}^{2+}$ traces and their raster plots are shown. (c) The frequency of glomerular spikes was drastically reduced $\left({ }^{* * *} \mathrm{p}<0.001\right.$, t-test) while the amplitude was marginally decreased (t-test, $\left.{ }^{*} \mathrm{p}=0.015\right)$. Error bars represent SD. More detailed analyses are in Figure S7. Data are from a total imaging time of 
598.88 and 571.64 minutes, from 10 and 8 imaging sessions from 2 and 3 animals for littermate controls and M/T-TeNT mice, respectively. (d) Primary dendrite pruning was significantly reduced in M/T-TeNT mice, at both P6 and P14. ${ }^{*} \mathrm{p}<0.05$, ${ }^{* * *} \mathrm{p}<0.001$ ( $\chi^{2}$-test). (e) Summary schema, highlighting the role of spontaneous network activity for the formation of parallel discrete connections in the olfactory bulb. Spontaneous activity generated within the olfactory bulb regulates the pruning of primary dendrites during Phase II. 


\section{METHODS}

\section{Mice.}

All animal experiments were approved by the Institutional Animal Care and Use Committee (IACUC) of the RIKEN Kobe Branch and Kyushu University. OMP-tTA BAC transgenic mice (OMP-tTA Tg; CDB0506T) and R26-TRE-GCaMP3 BAC transgenic mice (TRE-GCaMP3 Tg; CDB0505T) were generated in a previous study ${ }^{49}$. Pcdh21-Cre Tg (RBRC02189) ${ }^{72}, R 26-C A G-$ LoxP-TeNT knock-in (RBRC05154) ${ }^{73}$, floxed-Cnga $2^{74}$, floxed-Grin1 (JAX \#005246) ${ }^{75}$, OMPGFP knock-in (JAX \#006667) ${ }^{76}$, OMP-tTA knock-in (JAX \#17754) ${ }^{52}$, TRE-TeNT Tg (JAX \# 010713) ${ }^{77}$, OMP-Cre knock-in (JAX \#006668) ${ }^{78}$, a Cre-dependent GCaMP3 knock-in line Ai38 (JAX \#014538) ${ }^{54}$, Thy1-GCaMP6f Tg (line GP5.11) (JAX \#024339) ${ }^{53}$, Nkcc1 KO (MMRRC \#034262) ${ }^{79}$ have been described previously. ICR mice were used for in utero electroporation in Figure 1 and 2, and C57BL6N was used for Figure S1D. Grin1 ${ }^{f l / f l}$, OMP-Cre; Cnga ${ }^{f l / o}$, OMPCre;R26-CAG-LoxP-TeNT, Pcdh21-Cre;R26-CAG-LoxP-TeNT, Pcdh21-Cre;Ai38 in C57BL6 (C57BL6/N or C57BL/6J) or C57BL6 $\times 129$ (129P2 or 129S2) mixed background were bred to ICR mice for in utero electroporation (mixed at various ratios). Nkcc1 KO backcrossed to $\mathrm{FVB} / \mathrm{N}$ was bred to ICR mice for in utero electroporation (mixed at various ratios). Since the Cnga2 gene is X-linked, male hemizygous mutant mice (OMP-Cre;Cnga ${ }^{f l / o}$ ) were analyzed for Cnga2. For OSN-TeNT, we initially analyzed OMP-tTA knock-in mice ${ }^{52}$ crossed with TRETeNT Tg ${ }^{77}$; however, TeNT was not expressed in all OSNs and a substantial amount of VAMP2 immunoreactivity remained in the OSN axon terminals (data not shown). We therefore used OMP-Cre;R26-CAG-LoxP-TeNT (both are knock-in), in this case VAMP2 immunoreactivity completely disappeared in OSN axons (Figure 3D). When mixed genetic backgrounds were used for quantification, samples from littermates were used for control experiments. Mice used for $\mathrm{Ca}^{2+}$ imaging (OMP-tTA;TRE-GCaMP3, Pcdh21-Cre;Ai38, Thy1-GCaMP6f, Thy1GCaMP6f;OMP-Cre;R26-CAG-LoxP-TeNT, Thy1-GCaMP6f;Pcdh21-Cre;R26-CAG-LoxPTeNT) were in C57BL/6 or C57BL6 $\times 129$ mixed background. Both males and females were used for our experiments.

\section{Plasmid constructions.}

Cre-dependent pCA-LNL-GFP and Flp-dependent pCA-FNF-DsRed are kind gifts from C. Cepko (addgene \#13770 and \#13771) ${ }^{80}$. NaChBac is a kind gift from D. Clapham ${ }^{46}$. Kir2.1 (NM_008425.4) was PCR amplified from P10 mouse OB cDNA. Mutant Kir2.1 (mutKir2.1) ${ }^{45}$ was generated using PCR-mediated mutagenesis. TdTomato (a gift from R. Tsien) was PCRamplified and subcloned into pCA-LNL or pCA-FNF vector. Cre (addgene \#14797, a gift from C. Cepko), iCre (a gift from R. Sprengel), and FLPo (addgene \#13793, a gift from P. Soriano) were PCR-amplified and subcloned into pCAG vector. FLAG-tag was fused at the C-termini of Kir2.1, mutKir2.1, and NaChBac and subcloned into pCA-LNL vector. Dlg4 coding for PSD95 was PCR amplified from P10 mouse OB cDNA, and pCA-FNF-PSD $\Delta 1.2-$ GFP was generated as described previously ${ }^{42,43}$. We used PSD $\Delta 1$.2-GFP because the overexpression of full length PSD95 perturbed dendrite morphology in mitral cells (data not shown). Plasmids newly generated in this study will be deposited to Addgene (\#125573-125581).

\section{In utero electroporation.}

For sparse fluorescent labeling, pCA-LNL-tdTomato $(1 \mu \mathrm{g} / \mu \mathrm{L})$ and pCAG-Cre $(2-10 \mathrm{ng} / \mu \mathrm{L})$ 
were used. To label mutant neurons sparsely, pCA-FNF-tdTomato $(1 \mu \mathrm{g} / \mu \mathrm{L})$, pCAG-Flpo (2-10 $\mathrm{ng} / \mu \mathrm{L})$ were used. To generate single-cell Grin1 KO mutants, pCA-FNF-tdTomato $(1 \mu \mathrm{g} / \mu \mathrm{L})$, pCAG-Flpo (2-10 ng/ $\mu \mathrm{L})$, and pCAG-iCre $(1 \mu \mathrm{g} / \mu \mathrm{L})$ were used in homozygous floxed Grin1 mice. In utero electroporation was performed as described previously ${ }^{37}$. To label mitral cells at $\mathrm{E} 12,2 \mu \mathrm{L}$ of plasmid solutions were injected into the lateral ventricle and electric pulses (a single 10 -ms poration pulse at $72 \mathrm{~V}$, followed by five 50 -ms driving pulses at $32 \mathrm{~V}$ with 950 -ms intervals) were delivered along the anterior-posterior axis of the brain with forceps-type electrodes (3-mm diameter, LF650P3, BEX) and a CUY21EX electroporator (BEX). In utero electroporation for E10 embryos was performed as reported previously ${ }^{81}$.

\section{Naris occlusion.}

P0 mice were anesthetized on ice and a unilateral naris occlusion was quickly performed by cauterizing with soldering iron. Naris closure was confirmed under a stereomicroscope.

\section{Hand rearing.}

Hand rearing of newborn mice was performed as described previously (https://www.youtube.com/watch?v=sNX2byHbppM) with some modifications. Mice were kept in a boxed chamber (AsOne, \#AS-600SC) maintained at $28-30^{\circ} \mathrm{C}$ and a humidity of $50 \%$. A heater (Natsume, KN-475-3-40) and a water bath (Sansyo, SWS-181D) were used to maintain the temperature and humidity, respectively. Up to 3 pups were hand reared at the same time. Hand rearing was performed from P1 to P6. Pups were fed with Powdered Dog Milk at the recommended dilutions (Esbilac, PetAG, warmed to $37^{\circ} \mathrm{C}, \sim 50 \mu \mathrm{L} / \mathrm{g}$ ) with a paint brush every $\sim 2$ hours (done in shifts day and night). Their head and body were kept clean with a damp cotton bud. To facilitate digestion, excretion, and to prevent bloating, their abdomen was massaged with a cotton bud just after feeding. If the pups appeared dehydrated, a subcutaneous injection of saline was administered. A detailed step-by-step protocol will be published in Bio-protocols.

\section{Mouse brain samples.}

Mice were deeply anesthetized with an overdose i.p. injection of pentobarbital (Dainippon Sumitomo Pharma). Anesthetized mice were perfused with 4\% paraformaldehyde (PFA) in phosphate buffered saline (PBS). Excised brain samples were fixed with $4 \%$ PFA in PBS at $4{ }^{\circ} \mathrm{C}$ overnight. Samples were then embedded in 4\% low-melting agarose (Thermo Fisher, \#16520100) to prepare brain slices. For immunohistochemical analysis, samples were cryoprotected with $30 \%$ sucrose at $4{ }^{\circ} \mathrm{C}$ overnight and embedded in OCT compound (Sakura).

\section{Immunohistochemistry.}

Frozen OBs were cut into 16- $\mu$ m-thick coronal sections using a cryostat (Leica). Sections were blocked with 5\% normal donkey serum in PBS for 30 minutes and then incubated with mouse anti-VAMP2 (1:500, Synaptic Systems, 104211) in 5\% normal donkey serum at $4^{\circ} \mathrm{C}$ overnight. After washing 3 times in PBS, sections were incubated with Alexa 555-conjugated donkey antimouse IgG (1:500, ThermoFisher, A-31570) for 1 hour at room temperature. Nuclei were stained with DAPI. Images were taken by a confocal microscope (Olympus, FV1000).

\section{Optical clearing and imaging.}


Mouse brain slices (2 mm-thick for SeeDB, $500 \mu$ m-thick for SeeDB2) were cut using a microslicer (Dosaka EM). Slices were then cleared with SeeDB ${ }^{39,82}$ or SeeDB2 ${ }^{40}$ as described previously. Step-by-step protocol and technical tips have been described in SeeDB Resources (https://sites.google.com/site/seedbresources/). SeeDB2 samples were stained with DAPI. Cleared tissues were mounted on glass slides with 2-mm or $500 \mu \mathrm{m}$ silicone rubber spacer ${ }^{82}$. Samples were imaged with an upright two-photon microscope (Olympus, FV1000MPE) with a motorized stage (Sigma-Koki). A commercialized 25x objective lens (Olympus, XLPLN25XSVMP, NA 1.0, WD $4.0 \mathrm{~mm}$ ) were used to image SeeDB samples. InSight DeepSee Dual (Spectra-Physics) was used for two-photon excitation of tdTomato (1040 nm). SeeDB2 samples were imaged with inverted confocal microscopes: Olympus FV1000 with 405 nm, 488 nm, and 543 nm laser lines, or Leica SP8 with 405 nm and 552 nm laser lines.

\section{In vivo $\mathrm{Ca}^{2+}$ imaging of the olfactory bulb.}

Mouse lines. Transgenic OMP-tTA;TRE-GCaMP3(OSN-GCaMP3), Pcdh21-Cre;Ai38(M/TGCaMP3), or Thy1-GCaMP6f (GP5.11) (M/T-GCaMP6f) were used for in vivo $\mathrm{Ca}^{2+}$ imaging. For the imaging of mutant mouse strains they were crossed with the Thy1-GCaMP6f line (M/TGCaMP6f). It should be noted that robust odor-evoked responses ( $\Delta \mathrm{F} / \mathrm{F}>100 \%)$ were detected in the adult olfactory bulb using these mouse lines ${ }^{49}$. Odor responses were also seen in the neonates (data not shown).

Anesthesia. Neonatal mice were anesthetized with age and weight specific doses and concentrations of ketamine/xylazine that were administrated by intra-peritoneal injections. $10 \mathrm{mg} / \mathrm{ml}$ ketamine and $20 \mathrm{mg} / \mathrm{ml}$ xylazine and $\mathrm{H}_{2} \mathrm{O}$ were mixed and injected as below.

Ratios (ketamine/xylazine/ $\mathrm{H}_{2} \mathrm{O}$ in $\mathrm{mL}$ ) and doses (/g body weight):

P0: 10:0.5:21, 0.025 mL/g; P2: 10:0.5:21, 0.02 mL/g; P4: 10:0.5:6, 0.015mL/g; P6-16: 10:0.5:6, $0.01 \mathrm{~mL} / \mathrm{g} ; \mathrm{P} 20-30: 10: 0.5: 6,0.02 \mathrm{~mL} / \mathrm{g}$.

Surgery. Once anesthetized, a small craniotomy was made above the OB, and filled with KwikSil (WPI). Then a $2 \mathrm{~mm}$ circular coverslip (Matsunami) was placed above and sealed with superglue. Dental cement (Shofu) was then used to attach a custom-made bar (Narishige), allowing us to head fix with a custom head holding device (Narishige, MAG-2) and image once the mice had recovered.

\section{$\mathrm{Ca}^{2+}$ imaging of olfactory bulb slices in vitro.}

Pcdh21-Cre;Ai38(M/T-GCaMP3), or Thy1-GCaMP6f (GP5.11) (M/T-GCaMP6f) were used for in vitro $\mathrm{Ca}^{2+}$ imaging of olfactory bulb slices. Mouse pups were anesthetized on ice and sacrificed by decapitation. The olfactory bulb was quickly excised into ACSF (125 mM NaCl, 3 $\mathrm{mM} \mathrm{KCl}, 1.25 \mathrm{mM} \mathrm{NaH}_{2} \mathrm{PO}_{4}, 2 \mathrm{mM} \mathrm{CaCl}$, $1 \mathrm{mM} \mathrm{MgCl}_{2}, 25 \mathrm{mM} \mathrm{NaHCO}_{3}$, and $25 \mathrm{mM}$ glucose). OB slices were placed in a custom-made chamber. The chamber was placed under an upright two-photon microscope (Olympus, FV1000MPE) with a motorized stage (Sigma-Koki) and a water-immersion 25x objective lens (Olympus, XLPLN25XWMP, NA = 1.05, WD = 2.0). Before starting an imaging session, olfactory bulb slices are perfused with oxygenated ACSF (bubbled with $95 \% \mathrm{O}_{2}$ and $\left.5 \% \mathrm{CO}_{2}\right)$ at least for 1 hour at room temperature $\left(25^{\circ} \mathrm{C}\right)$. During 
imaging sessions, olfactory bulb slices were continuously perfused with oxygenated ACSF $(0.85-1.07 \mathrm{~mL} / \mathrm{min})$ at room temperature.

\section{Pharmacology.}

$50 \mu \mathrm{M}$ APV (Sigma, A5282), $25 \mu \mathrm{M}$ CNQX (Sigma, C239), $300 \mu \mathrm{M}$ Carbenoxolone (Sigma, C4790), 1mM 1-octanol (Sigma, 95446), $100 \mu$ M GABA (Sigma, A5835), $1 \mu$ M TTX (Abcam, ab120055), were used for pharmacological experiments for olfactory bulb slices.

\section{Quantification of dendrites.}

Only the medial side of the olfactory bulb was analyzed. We only analyzed neurons located in the mitral cell layer. Therefore, external tufted cells and granule cells, sometimes labelled by in utero electroporation, were excluded from our analysis. We only quantified neurons where the entire dendritic field was included in the 3D image blocks. In our SeeDB protocol, glomeruli could be identified by autofluorescence signals. All primary dendrites were manually traced with Neurolucida software (MBF Bioscience). Feeding and quantification of dendrites were blinded in the hand rearing experiments. In all the remaining experiments, quantification was not blinded; however, to exclude any biases, all labelled neurons that meet the above criteria were quantified in an unbiased manner. Littermates were used as controls. Criteria for quantification are described in Figure 2B.

\section{Analysis of in vivo $\mathrm{Ca}^{2+}$ imaging data.}

Motion and Drift Correction. Motion correction and drift correction were performed by using TurboReg (translation only, http://bigwww.epfl.ch/thevenaz/turboreg) then SPM (www.fil.ion.ucl.ac.uk/spm). Noise removal and subsequent $\Delta \mathrm{F} / \mathrm{F}$ movies were performed using AFID (a MATLAB plugin created by Dr. A. Lowe, https://github.com/mleiwe/AFID) ${ }^{83}$.

Spike Detection. Glomeruli were manually selected and $\Delta \mathrm{F} / \mathrm{F}$ values were calculated for each glomerulus. To detect glomerular spikes, a normal distribution was fitted to the lowest $80 \%$ of data points. Glomerular spikes were defined as points that were 3 standard deviations above the mean value calculated from a 10 second window around the time point in question.

Correlation Index. In order to measure the correlation of glomeruli a new measurement was created that measured the number of glomeruli active per frame $\left(G^{n}\right)$ as a percentage of the mean number of active glomeruli per frame $\left(\overline{G_{A}}\right)$. This normalization was necessary in order to control for differences in the number of glomeruli imaged as well as differences in the firing frequency of the network.

$$
\text { Correlation Index }=\frac{G^{n}}{\overline{G_{A}}} \times 100
$$

$H$ - and L-events. $\mathrm{H} / \mathrm{L}$ events were determined by calculating the number of glomeruli that were active in each frame. In Figures 4e,f,g, 7 and Supplementary Figure 4a, 7b, if the correlation index was above $5 \%$, and contained two or more glomeruli it was classed as an H-event. 
Otherwise, if there were events present it was classed as an L-event.

Spike Timing Tiling Coefficient (STTC) analysis. Calculations were performed as described previously ${ }^{59}$. Correlations were counted within a 2 second window (i.e. $\Delta \mathrm{T}=2$ seconds).

Custom written MATLAB codes were used for all in vivo and in vitro analyses and are available at GitHub (https://github.com/mleiwe/SpontActivityOfDevMitralCells).

\section{Analysis of slice $\mathrm{Ca}^{2+}$ imaging data.}

$\mathrm{Ca}^{2+}$ imaging data were processed by using ImageJ (NIH) and MATLAB (Mathworks). Glomeruli were manually selected as polygonal region of interests (ROIs) on ImageJ. ROI information is converted to region mask using poly2mask function. Average fluorescence intensity within each ROI is defined as $\mathrm{F}_{\text {. }} \mathrm{F}_{0}$ is defined as the average of 10-30 percentile values of fluorescence intensity in each glomerulus. $\Delta \mathrm{F} / \mathrm{F}$ values were calculated for each glomerulus.

$$
\Delta \mathrm{F} / \mathrm{F}=\left(\mathrm{F}-\mathrm{F}_{0}\right) / \mathrm{F}_{0} \times 100
$$

Motion correction and drift correction were not performed for in vitro imaging data analysis. Baselines were corrected by polynomial curve fitting. Raster plots were plotted by detecting peak points located in more than 4 standard deviations above the baselines. For calculation of standard deviations, top 25 percentile data were excluded. Correlation Index, H- and L- events were determined as described above. Cross correlation was calculated from $\Delta \mathrm{F} / \mathrm{F}$. The value was normalized so that the autocorrelations at zero lag equal 1. $C_{\max }$ and $T_{\text {latency }}$ were defined as shown in Supplementary Figure 5b. Cross correlation matrix shows $C_{\max }$ values of all combinations of two glomeruli. Average frequency and amplitude were calculated from the number of peak points and $\Delta \mathrm{F} / \mathrm{F}$ value at each peak point, respectively.

\section{GCaMP images and movies.}

GCaMP fluorescence images shown in Figure 4, 5, 7, and Supplementary Figure 3, 7 are maximum intensity projection images throughout the imaging session (also background subtracted) showing glomeruli. To prepare serial GCaMP images and $\triangle F / F$ images, spatial median filter (diameter, 3 pixels) was applied to reduce the shot noise. For $\Delta F / F$ images, $F 0$ values were created from the lowest $80 \%$ of pixel values. $\triangle F / F$ images are shown in pseudocolor (inferno). Unprocessed raw image data will be deposited to SSBD database (http://ssbd.qbic.riken.jp/set/2019xxxx/).

\section{Statistical analysis.}

Excel, Prism7, and MATLAB were used for statistical analysis. Sample sizes were not predetermined. However, post-hoc power analyses determined that sufficient samples were collected. All the statistical data including the sample size and the statistical test used in each figure panel are described in Supplementary Table 1. The number of neurons is described within figures, and all the numerical data including the numbers of animals are in Supplementary Table 2. Number of animals used for $\mathrm{Ca}^{2+}$ imaging was at least three and indicated within figures. $\chi^{2}$-tests were used in Figure 2b,c, 3a,b,e,j, 6e,f, 7d, and 
Supplementary Figure 2c,d,f, 6c. One-way ANOVA with Tukey post hoc tests were used in Figure 4. Wilcoxon signed-rank test was used in Figure 6a-c, and Supplementary Figure 6a. Kruskal-Wallis test and post hoc Dunn's multiple comparison test was used in Figure 1d.

Welch's t-test was used in Figure 3c, 3f, 5d, and 7a. Welch's t-tests, or Mann Whitney U tests were performed for imaging data in Figures 7c and Supplementary Figure 7b. In box plots, the middle bands indicate the median; boxes indicate the first and third quartiles; and the whiskers indicate the minimum and maximum values. Data inclusion/exclusion criteria are described in figure legends.

\section{Data and code availability.}

Raw image data used in this study will be deposited to Systems Science of Biological Dynamics (SSBD) database (http://ssbd.qbic.riken.jp/set/2019xxxx/). Neurolucida tracing data will be deposited to NeuroMorpho.Org (http://neuromorpho.org/). Numerical data for all graphs are included in Supplementary Table 2. Program codes have been deposited to GitHub (https://github.co.jp/) as detailed above. Requests for additional program codes and data generated and/or analyzed during the current study should be directed to and will be fulfilled on reasonable request by the Lead Contact, Takeshi Imai (t-imai@med.kyushu-u.ac.jp). 
Supplementary Information

\title{
Spontaneous activity generated within the olfactory bulb establishes discrete olfactory connections
}

\author{
Satoshi Fujimoto ${ }^{1,2,8}$, Marcus N. Leiwe ${ }^{1,2,8}$, Richi Sakaguchi ${ }^{1,2,3}$, Yuko \\ Muroyama $^{4}$, Reiko Kobayakawa ${ }^{5}$, Ko Kobayakawa ${ }^{5}$, Tetsuichiro Saito ${ }^{4}$, \& \\ Takeshi Imai ${ }^{1,2,3,6,7,9 *}$
}

Correspondence should be addressed to T.I. (t-imai@med.kyushu-u.ac.jp)

Supplementary Table 1, 2

Supplementary Figure 1-7

Supplementary Video 1-5 


\section{Supplementary Tables}

\section{Supplementary Table 1}

Statistics data reporting by figure (Excel file).

\section{Supplementary Table 2}

Numerical data for figure panels (Excel file). Each tab contains the numerical data for each figure panel. 


\section{Supplementary Figures}

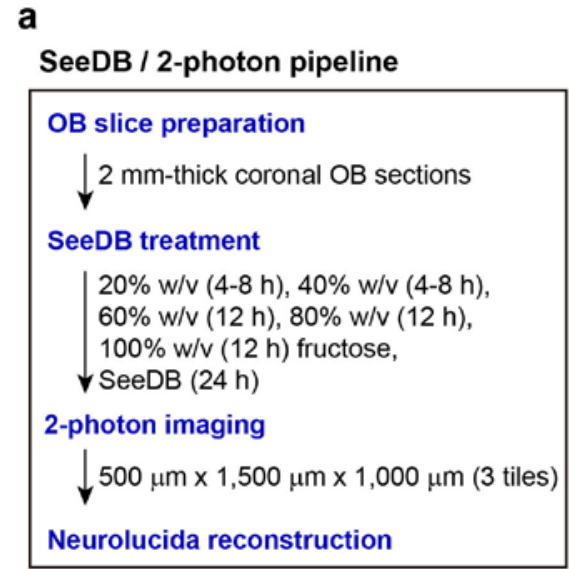

a

SeeDB / 2-photon pipeline
\begin{tabular}{|l|}
\hline OB slice preparation \\
$\downarrow 2 \mathrm{~mm}$-thick coronal OB sections \\
SeeDB treatment \\
$\quad 20 \% \mathrm{w} / \mathrm{v}(4-8 \mathrm{~h}), 40 \% \mathrm{w} / \mathrm{v}(4-8 \mathrm{~h})$, \\
$60 \% \mathrm{w} / \mathrm{v}(12 \mathrm{~h}), 80 \% \mathrm{w} / \mathrm{v}(12 \mathrm{~h})$, \\
$100 \% \mathrm{w} / \mathrm{v}(12 \mathrm{~h})$ fructose, \\
$\downarrow$ SeeDB $(24 \mathrm{~h})$ \\
2-photon imaging \\
$\downarrow 500 \mu \mathrm{m} \times 1,500 \mu \mathrm{m} \times 1,000 \mu \mathrm{m}(3$ tiles $)$ \\
Neurolucida reconstruction \\
\hline
\end{tabular}

SeeDB2 / confocal pipeline

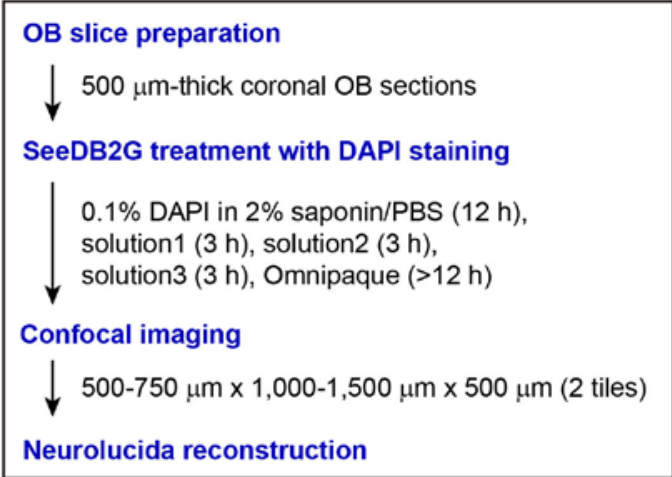

b

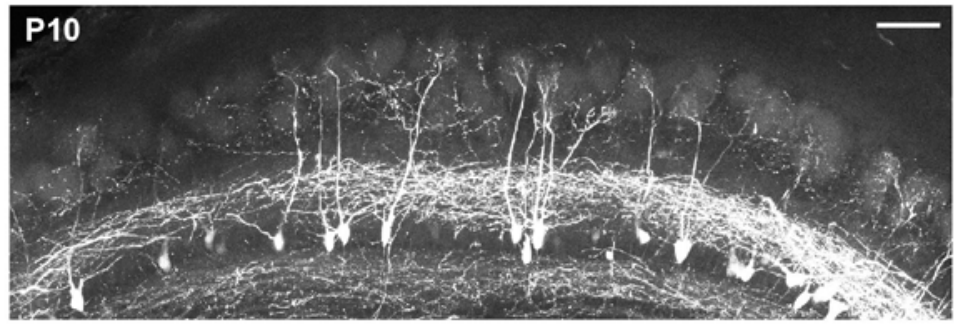

C

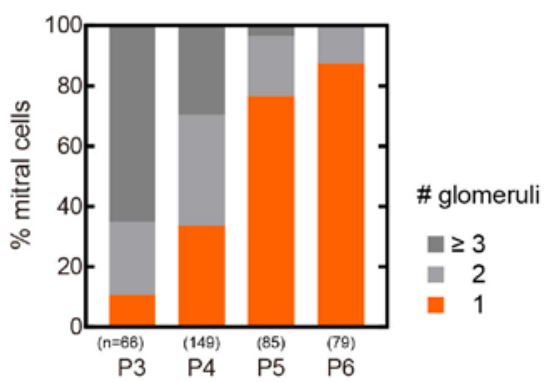

\section{Supplementary Figure 1}

Quantitative analyses of mitral cell dendrites. (a) The clearing and imaging pipeline. OB slices were cleared with SeeDB or SeeDB2 and imaged using two-photon microscopy (excitation at $1040 \mathrm{~nm}$ for tdTomato) or confocal microscopy (excitation at 405nm for DAPI and $552 \mathrm{~nm}$ for tdTomato), respectively. (b) An example of mitral cells reconstructed by SeeDB-cleared olfactory bulb samples (100 $\mu \mathrm{m}$ stack) (see also Supplementary Video 1 for 1mm-thick images). The medial side of the OB was imaged by two-photon microscopy and all the labelled mitral cells were quantified in an unbiased manner. Autofluorescence signals were used to identify glomeruli for SeeDB samples. DAPI signals were used to identify glomeruli for SeeDB2 samples. The scale bar represents $100 \mu \mathrm{m}$. (c) Day-to-day quantifications of primary dendrites in C57BL/6N mice. 


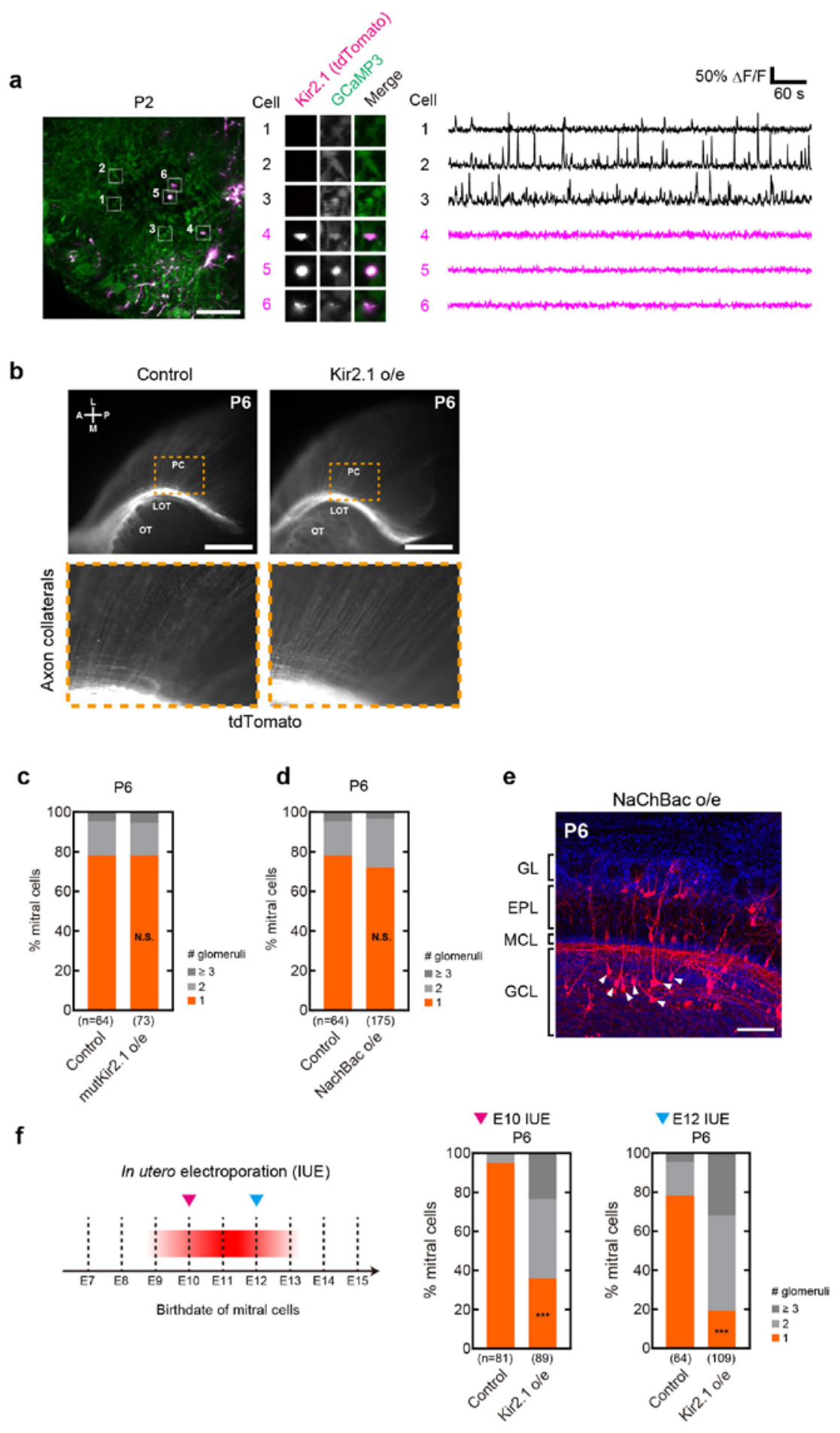

\section{Supplementary Figure 2}

Genetic manipulations of neuronal activity in mitral cells. (a) Spontaneous activity was not detected in Kir2.1-expressing mitral cells at P2. Kir2.1 was introduced to mitral cells in the M/TGCaMP3 mice using in utero electroporation. Scale bar, $100 \mu \mathrm{m}$. Imaging was performed at $0.429 \mathrm{sec} /$ frame. (b) Axonal projections of mitral cells expressing tdTomato alone (control, left panels) or with Kir2.1 (right panels). No obvious differences were seen in either the axonal extension in the lateral olfactory tract (LOT) and collateral formation in the piriform cortex (PC), 
at least at P6. OT, olfactory tubercle. Scale bar represents $1 \mathrm{~mm}$. (c) Mitral cells expressing mutant Kir2.1 (mutKir2.1), a non-conducting mutant of the Kir2.1 potassium channel. No defect in the pruning process was observed $\left(\chi^{2}\right.$-test), indicating that the defective dendrite pruning by Kir2.1 overexpression is due to its hyperpolarizing effects. The control is the same as shown in Figure 2b. (d) Mitral cells expressing NaChBac, the bacterial sodium channel. It has been reported that $\mathrm{NaChBac}$ expression increases the resting membrane potential and thereby increases the firing frequency in neurons. However, no significant differences were seen $\left(\chi^{2}\right.$-test). The control is the same as shown in Figure 2b. (e) NaChBac-expressing neurons showed dispositioning of cell body; somata were frequently located beneath the mitral cell layer (arrowheads). These dispositioned mitral cells were excluded from the quantification in (d). (f) Quantification of dendrite pruning for early-born mitral cells. In utero electroporation was performed for E10 embryos to label early-born mitral cells. The results were similar to E12labelled ones (right, same data as shown in Figure $\mathbf{2 b}$ ). 
a

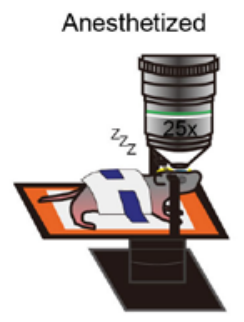

C
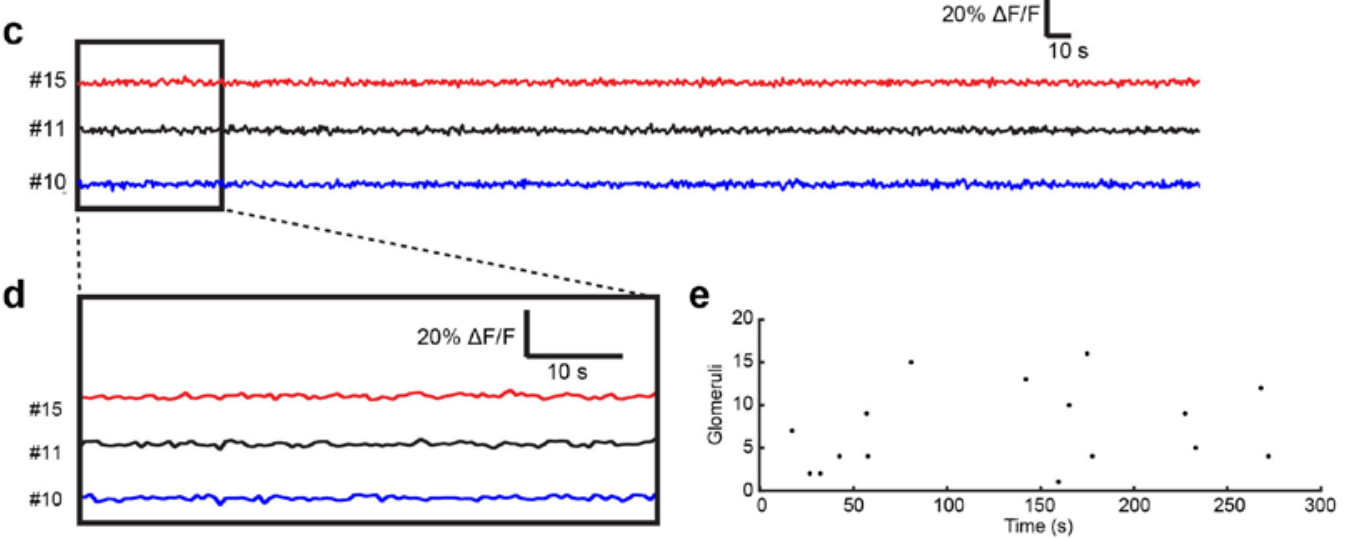

f OSN-TeNT (Maternally reared, P6)
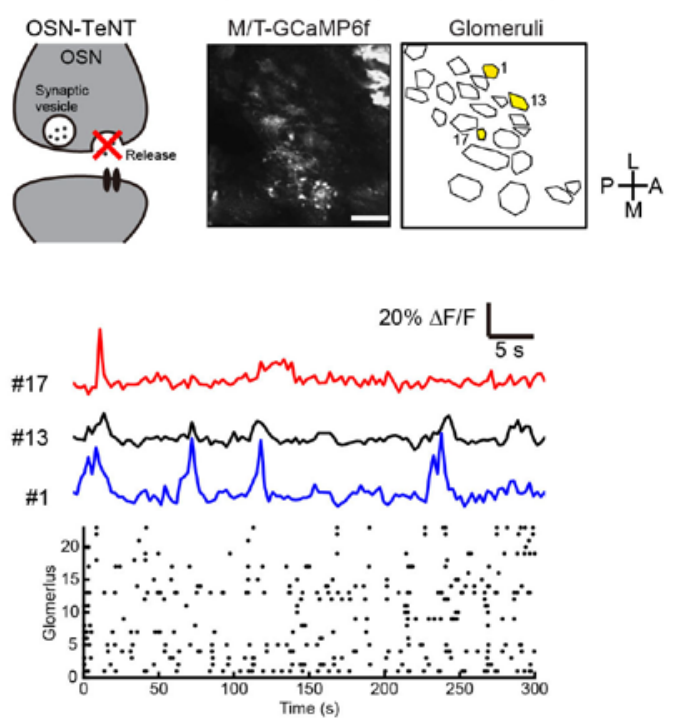

\section{Supplementary Figure 3}

Additional in vivo $\mathrm{Ca}^{2+}$ imaging of the olfactory bulb. (a) Schematic display of the anaesthetized set up. (b) A fluorescence image (maximal intensity image throughout the session) and corresponding ROI selection for example traces. (c) Example traces for yellow ROIs in (B) showing almost no spontaneous activity. (d) Zoomed in area displays that the glomerular spikes are not evident. (e) Raster plot displaying the spikes detected over a five minute imaging period in the anesthetized mouse. (f) Imaging of OSN-TeNT mice crossed with M/T-GCaMP6f mice shows that spontaneous activity remains in mitral cells. Scale bars, $100 \mu \mathrm{m}$. 
a $\mathrm{P} 1$
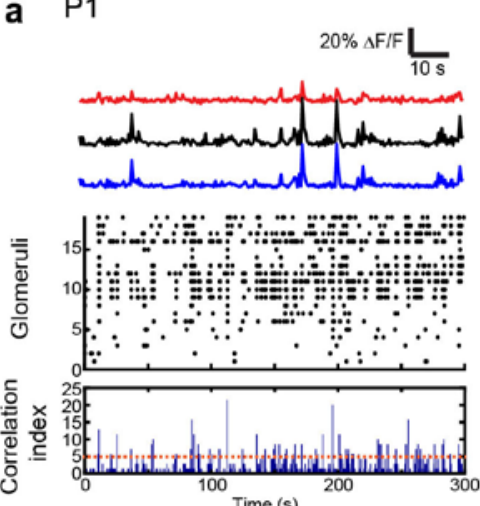

P4
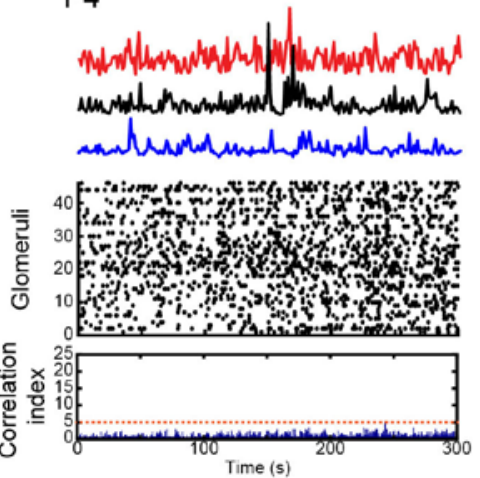

b

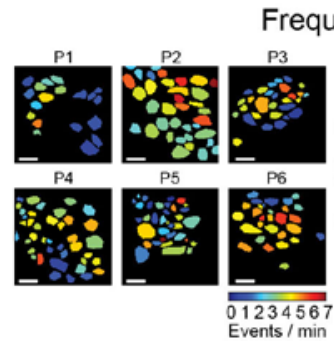

d

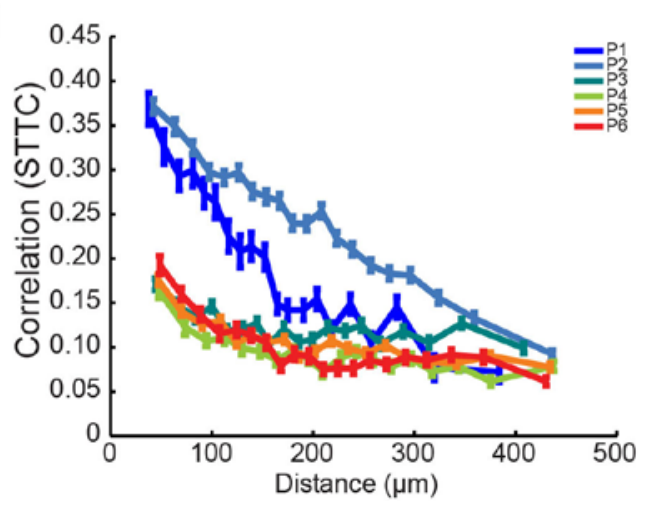

P2

P5

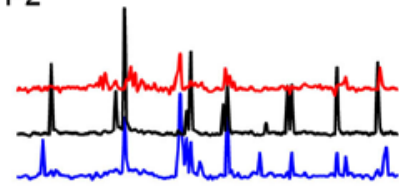

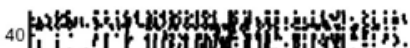

30 2 s?

20 in

10 id
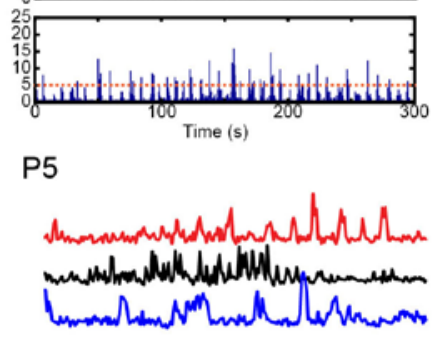

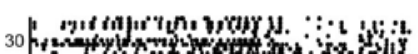

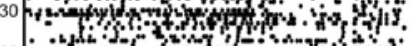

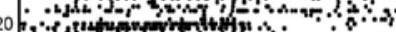

: Af
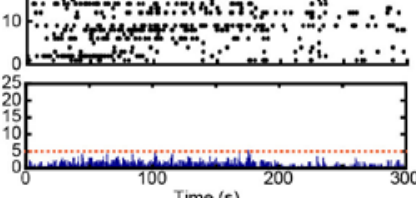

C

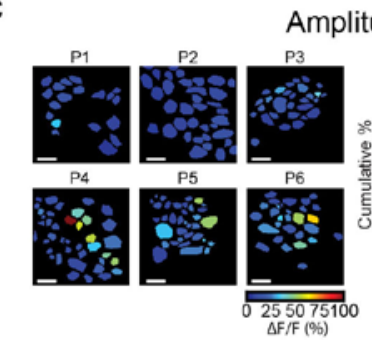

Amplitude
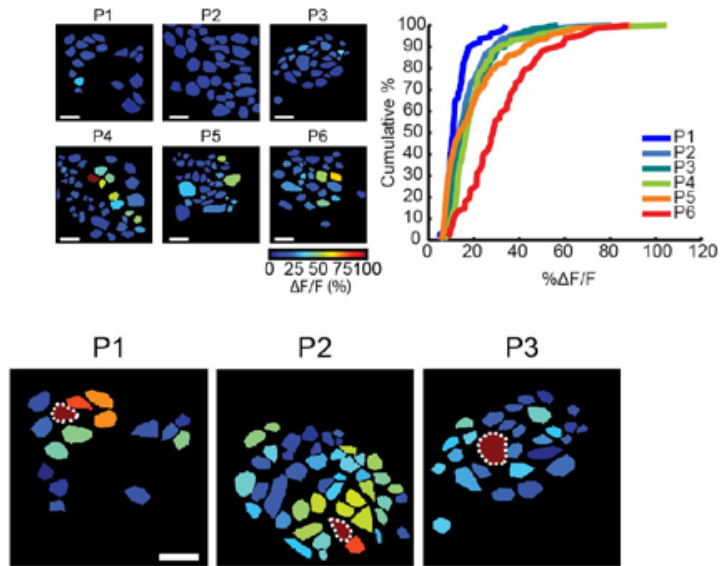

P4
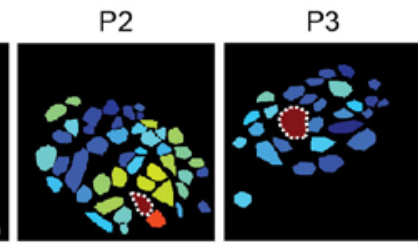

P5

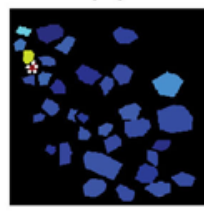

P6

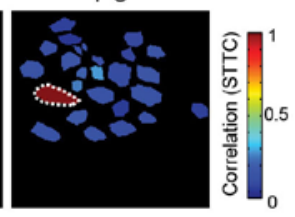

\section{Supplementary Figure 4}

Developmental changes of spontaneous activity in vivo. (a) Representative traces, raster plots, and correlation index histograms (P1-6). Orange dotted lines indicate the threshold value for $\mathrm{H}$ and L-events. P2 and P6 data are the same as in Figure 4. (b) Representative distributions of glomerular firing frequencies (P1-6, left). Cumulative distribution plots for the firing frequency 
of glomeruli (right). A data point represents the average firing frequency of a glomerulus over the imaging session. Data are for 77, 365, 210, 46, 394, and 222 glomeruli from 2, 4, 3, 3, 3, and 3 animals for ages P1-6, respectively. (c) Representative distributions of glomerular spike amplitude (P1-6, left). Cumulative distribution plots for the mean amplitude of glomerular spikes across all ages (right). A data point represents the average amplitude of spikes from a single glomerulus during an imaging session. (d) Spike Timing Tiling Coefficient (STTC) analysis shows the spatio-temporal relationship of the activity. P1 and P2 spontaneous activity showed a higher correlation within 100-150 $\mu \mathrm{m}$, while later stages did not. Data points represent the mean \pm SEM of glomerular comparisons into 20 groups, derived from 3, 11, 7, 3, 11, and 7 imaging sessions from 2, 4, 3, 3, 3, and 3 animals with total imaging times of 50.01, 211.46, 127.07, 57.87, 235.13, and 126.48 minutes for P1-P6 pups, respectively. Representative example heat maps for an example glomerulus (encircled by white dotted lines) are shown (right). Scale bars, $100 \mu \mathrm{m}$. 
a
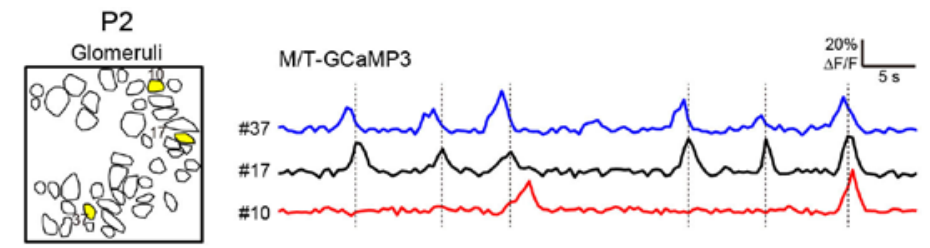

b

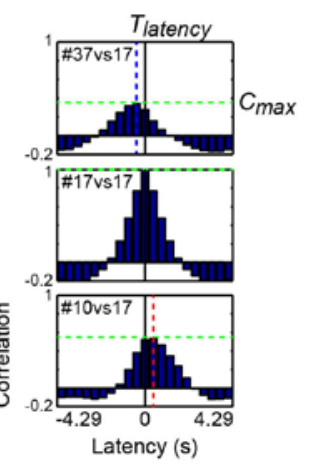

$C_{\text {max }}=0.536$

$T_{\text {latency }}=0.429$

$c_{\max }=1$

$T_{\text {latency }}=0$

$C_{\text {max }}=0.342$

$T_{\text {latency }}=-0.429$

C

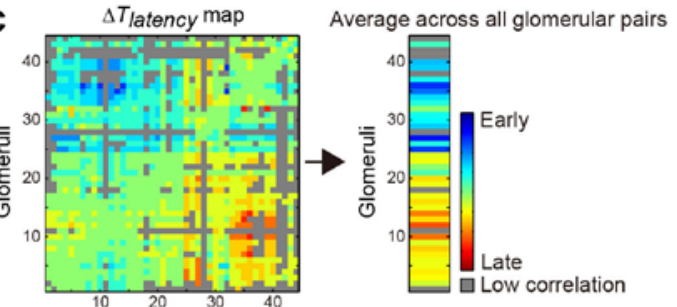

h

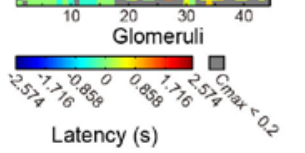

d
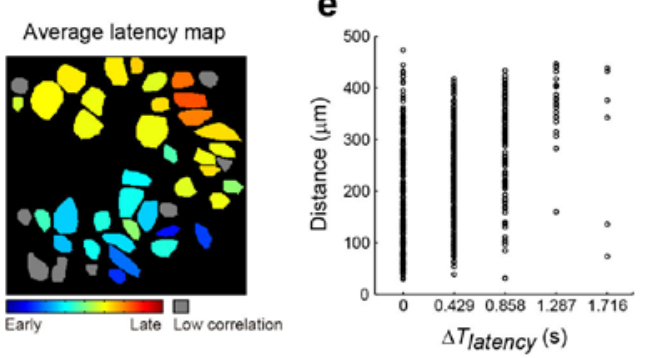

f

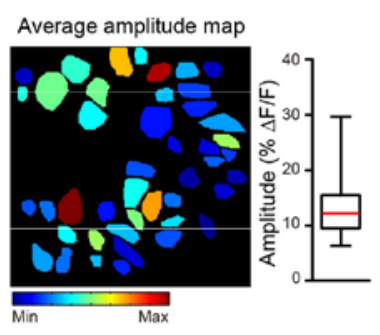

g
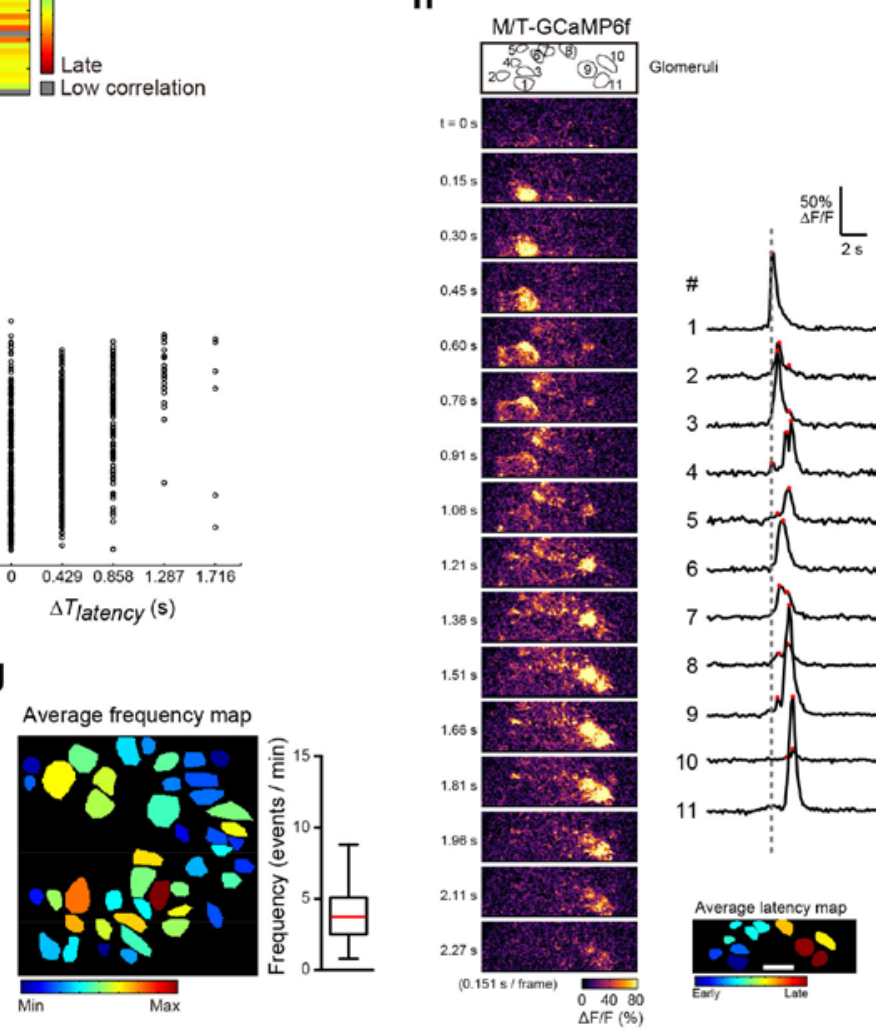

Supplementary Figure 5

Spatiotemporal patterns of spontaneous activity at P2 in vitro. (a, b) Calculation of the maximum value of cross correlation $\left(C_{\max }\right)$ and latency $\left(T_{\text {latency }}\right)$ to reach the $C_{\max }$. Raw traces (a) and cross correlograms (b) of 3 representative glomeruli (yellow ROIs) are shown.

(c) $\Delta T_{\text {latency }}$ map and average latency colormap. Glomerular pairs whose $C_{\max }$ values are smaller than 0.2 are shown in gray in the $\Delta T_{\text {latency }}$ map. Average latency was calculated based on the 
$\Delta T_{\text {latency }}$ map. Data were excluded from analysis when $C_{\text {max }}$ values were $<0.2$ in more than half of glomerular pairs (shown in gray). (d) A latency map generated from the P2 olfactory bulb slice (shown in Figure 5B) demonstrates a propagating wave at this stage. The average latency was determined as in (c). However, the average latency was mosaically arranged at a local scale. (e) The $\Delta T_{\text {latency }}$ and distance are plotted for all glomerular pairs. Glomerular pairs with $C_{\max }<0.2$ were excluded from this analysis. Based on this result, the propagation speed is estimated to be around 100-300 $\mu \mathrm{m} / \mathrm{sec}$ in vitro. Note that in vitro experiments were performed at room temperature. (f, g) Average amplitudes (f) and frequencies (g) of glomerular spikes for P2 olfactory bulb slice (shown in Figure $5 \mathbf{b}$ ). (h) High-speed $\mathrm{Ca}^{2+}$ imaging of olfactory bulb slices using M/T-GCaMP6f. Representative serial $\Delta \mathrm{F} / \mathrm{F}$ images of a propagating activity at $\mathrm{P} 2$ are shown. $\mathrm{Ca}^{2+}$ traces of individual glomeruli, show a propagating wave. Imaging was performed at $0.151 \mathrm{sec} /$ frame. The average latency map is shown. Scale bar, $100 \mu \mathrm{m}$. 
a
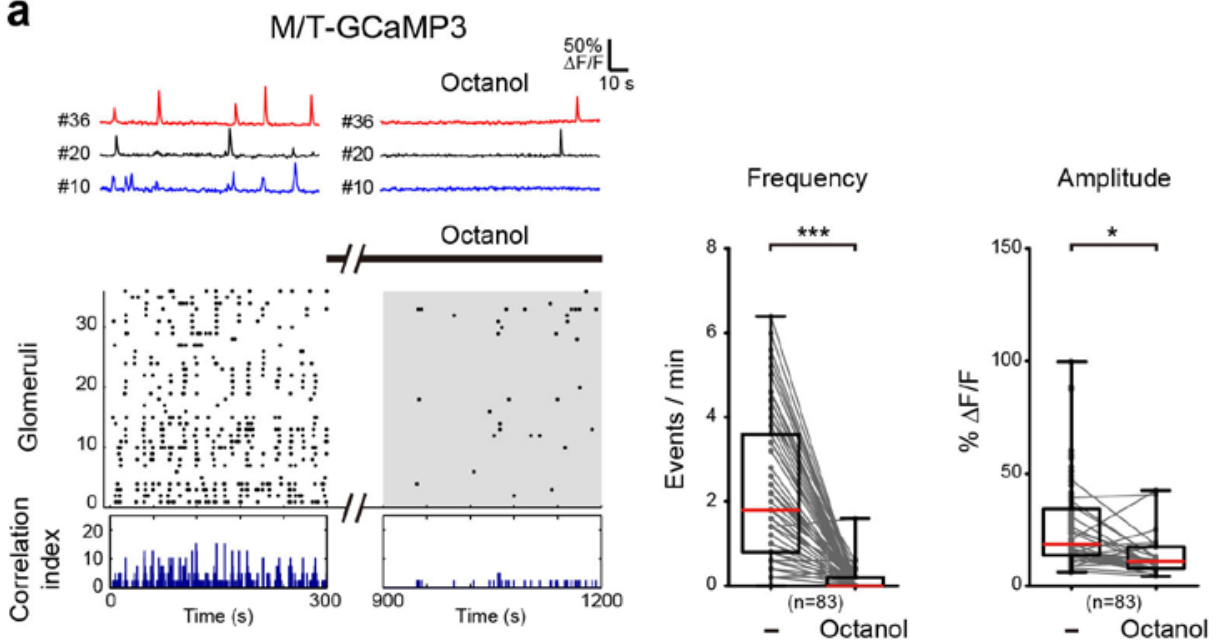

b

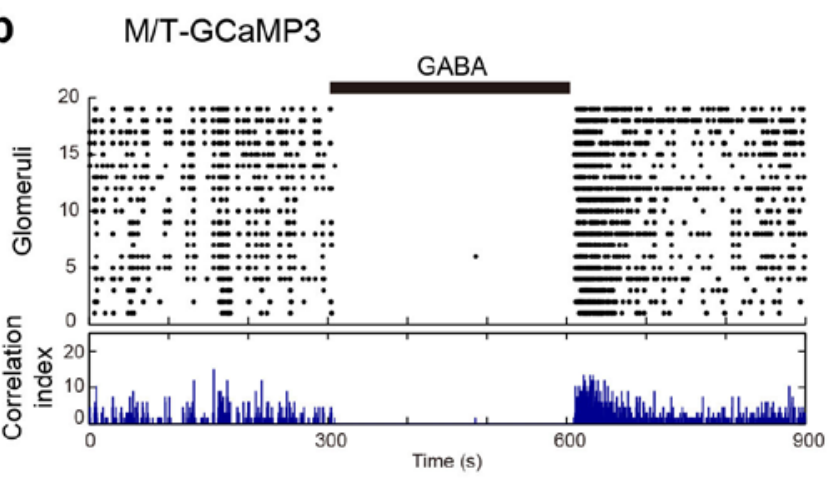

C

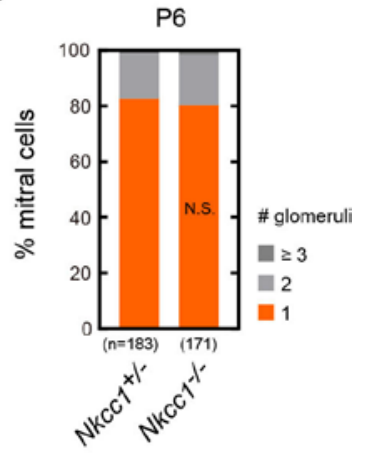

\section{Supplementary Figure 6}

Roles of gap junctions and GABA for spontaneous activity. (a) $\mathrm{Ca}^{2+}$ traces, raster plots, and correlation index scores before and after Octanol (1 mM) application (P2) (left). Firing frequencies, but not amplitudes, were reduced (right). $n=83$ glomeruli from 3 animals. (b) GABA $(100 \mu \mathrm{M})$ was applied to P2 olfactory bulb slices. Spontaneous activity in mitral cells was almost completely suppressed by GABA, suggesting an inhibitory role for GABA in mitral cells at this stage. (c) A knock-out mouse for NKCC1 did not show any defects in dendrite pruning in mitral cells. NKCC1 is a $\mathrm{Na}^{+}-\mathrm{K}^{+}-2 \mathrm{Cl}^{-}$cotransporter essential to incorporate $\mathrm{Cl}^{-}$ion into cells and to make the neuron excitatory to GABA. N.S., non-significant $\left(\chi^{2}\right.$-test compared to $\mathrm{NkCC1}^{+--}$). 
a

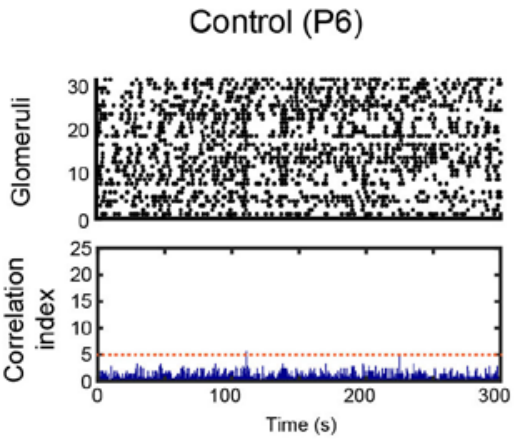

M/T-TeNT (P6)
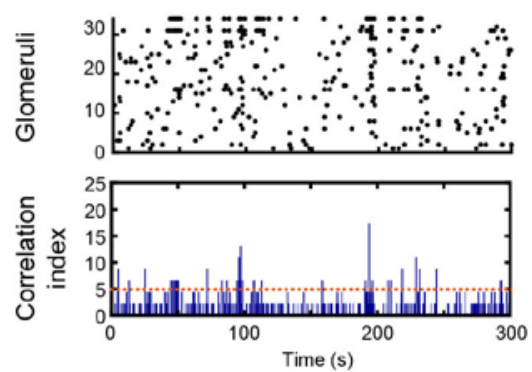

b
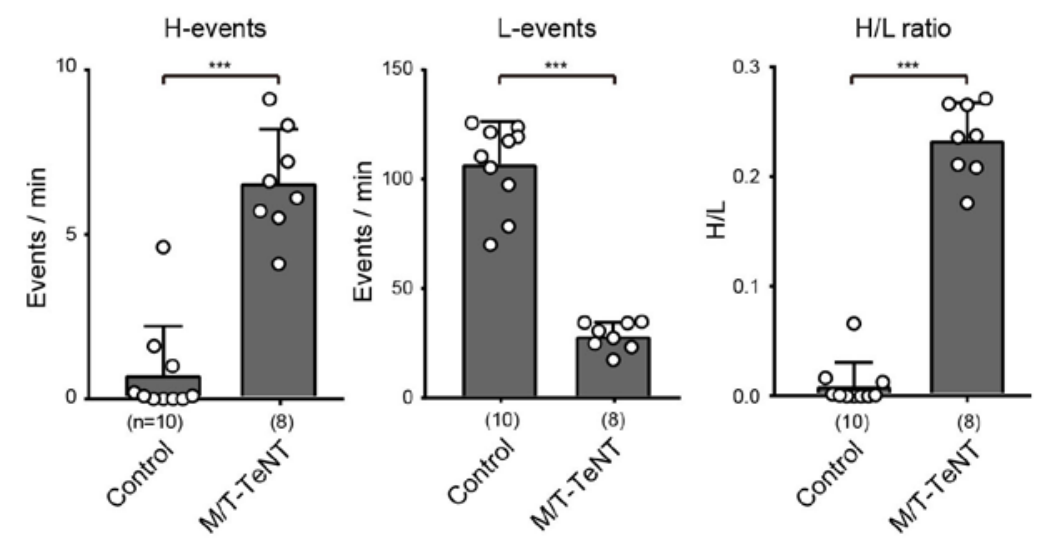

\section{Supplementary Figure 7}

Patterns of spontaneous activity in M/T-TeNT mice. (a) Raster plots (the same as in Figure 7b), and correlation index scores for control (littermates, left) and M/T-TeNT (right) mice. (b) Measurements of $\mathrm{H}$ - and L-events show increased $\mathrm{H} / \mathrm{L}$ ratio in $\mathrm{M} / \mathrm{T}$-TeNT mice. $\mathrm{N}$ values are identical to those listed in Figure 7. Error bars represent the SD. 


\section{Supplementary Videos}

\section{Supplementary Video 1}

Sparse labeling and optical clearing of OB. Mitral cells were sparsely labelled with tdTomato using in utero electroporation and then the olfactory bulb was cleared with SeeDB. Glomeruli can be identified with autofluorescence. All the labelled neurons were quantified in an unbiased manner.

\section{Supplementary Video 2}

Spontaneous activity in vivo at P2. P2 olfactory bulb was imaged using two-photon microscopy. An awake M/T-GCaMP6f mouse was analyzed. Raw fluorescence (F) and $\Delta \mathrm{F} / \mathrm{F}$ images are shown.

\section{Supplementary Video 3}

Spontaneous activity in vivo at P6. P6 olfactory bulb was imaged using two-photon microscopy. An awake M/T-GCaMP6f mouse was analyzed. Raw fluorescence (F) and $\Delta F / F$ images are shown.

\section{Supplementary Video 4}

Spontaneous activity in vitro at P2. P2 olfactory bulb was imaged in vitro using two-photon microscopy. An M/T-GCaMP3 mouse was analyzed. Raw fluorescence (F) and $\Delta \mathrm{F} / \mathrm{F}$ images are shown.

\section{Supplementary Video 5}

Spontaneous activity in vitro at P6. P6 olfactory bulb was imaged in vitro using two-photon microscopy. An M/T-GCaMP3 mouse was analyzed. Raw fluorescence (F) and $\Delta \mathrm{F} / \mathrm{F}$ images are shown. 